\title{
QUENCHED INVARIANCE PRINCIPLE FOR LONG RANGE RANDOM WALKS IN BALANCED RANDOM ENVIRONMENTS
}

\author{
XIN CHEN ZHEN-QING CHEN TAKASHI KUMAGAI JIAN WANG
}

\begin{abstract}
We establish via a probabilistic approach the quenched invariance principle for a class of long range random walks in independent (but not necessarily identically distributed) balanced random environments, with the transition probability from $x$ to $y$ on average being comparable to $|x-y|^{-(d+\alpha)}$ with $\alpha \in(0,2]$. We use the martingale property to estimate exit time from balls and establish tightness of the scaled processes, and apply the uniqueness of the martingale problem to identify the limiting process. When $\alpha \in(0,1)$, our approach works even for non-balanced cases. When $\alpha=2$, under a diffusive with the logarithmic perturbation scaling, we show that the limit of scaled processes is a Brownian motion.
\end{abstract}

Keywords: long range random walk; balanced random environment; martingale problem

MSC 2010: 60G51; 60G52; 60J25; 60J75.

\section{INTRODUCTION AND MAIN RESULT}

Let $(\Omega, \mathscr{F}, \mathbb{P})$ be a probability space. We will use $\mathbb{E}$ to denote the mathematical expectation taken under $\mathbb{P}$. Let $\mathbb{Z}$ and $\mathbb{Z}_{+}$denote the set of integers and non-negative integers, respectively. For $d \geqslant 1$, let $\mathbb{Z}^{d}$ be the $d$-dimensional integer lattice, and $\mathbb{Z}_{\mathbf{0}}^{d}:=\mathbb{Z}^{d} \backslash\{\mathbf{0}\}$, where $\mathbf{0}:=(0, \cdots, 0)$ is the zero element in $\mathbb{Z}^{d}$ (or $\mathbb{R}^{d}$ ). Here and in what follows, we use $:=$ as way of definition. For each fixed $\omega \in \Omega$, we consider a continuous time random walk $X^{\omega}:=\left(X_{t}^{\omega}\right)_{t \geqslant 0}$ on $\mathbb{Z}^{d}$ with the infinitesimal generator

$$
\mathcal{L}^{\omega} f(x):=\sum_{z \in \mathbb{Z}_{\mathbf{0}}^{d}}(f(x+z)-f(x)) \frac{\kappa(x, z)(\omega)}{|z|^{d+\alpha}}, \quad f \in B_{b}\left(\mathbb{Z}^{d}\right),
$$

where $0<\alpha \leqslant 2$ and $\kappa(x, z)(\omega)$ satisfies the following balanced condition

$$
\kappa(x, z)(\omega)=\kappa(x,-z)(\omega) \geqslant 0 \quad \text { for all } x \in \mathbb{Z}^{d}, z \in \mathbb{Z}_{\mathbf{0}}^{d} \text { and } \omega \in \Omega .
$$

In other words, $X^{\omega}$ is a continuous time Markov process on $\mathbb{Z}^{d}$ such that conditioned on $X_{t}^{\omega}=x, X^{\omega}$ waits an exponentially distributed random amount of time with parameter

$$
\lambda^{\omega}(x):=\sum_{z \in \mathbb{Z}_{\mathbf{0}}^{d}} \kappa(x, z)(\omega)|z|^{-(d+\alpha)}
$$

before it jumps to $x+z$ with probability $\kappa(x, z)(\omega)|z|^{-(d+\alpha)} / \lambda^{\omega}(x)$. When $\alpha \in(0,2)$ and $\kappa(x, z)(\omega)$ is uniformly elliptic, i.e., there exist (non-random) constants $0<c_{1} \leqslant c_{2}<\infty$ such that $c_{1} \leqslant \kappa(x, z)(\omega) \leqslant$ $c_{2}$ for all $x \in \mathbb{Z}^{d}, z \in \mathbb{Z}_{\mathbf{0}}^{d}$, and $\omega \in \Omega$, the process $X^{\omega}$ is called an $\alpha$-stable-like (balanced but not necessarily symmetric) random walk in the literature.

If $\kappa(x, z)(\omega)=0$ for all $\omega \in \Omega, x \in \mathbb{Z}^{d}$ and $z \in \mathbb{Z}^{d}$ with $|z|>1$, then the process $X^{\omega}$ is reduced into a nearest neighbor random walk in balanced random environments (NNBRW). In particular, a NNBRW can be viewed as the discrete counterpart corresponding to an $\mathbb{R}^{d}$-valued diffusion process in balanced random environments, which was initially considered by Papanicolaou and Varadhan [13]. The NNBRW was first introduced by Lawler [12], where the quenched invariance principle was established under some uniformly elliptic condition on the conductance in ergodic environments. Thirty years later, Guo and Zeitouni [11] proved the quenched invariance principle for NNBRWs under some inverse moment condition on the conductance in balanced random environments. When the environment is i.i.d., (that is, $\{\kappa(x, \cdot)\}_{x \in \mathbb{Z}^{d}}$ are i.i.d. across the sites $x \in \mathbb{Z}^{d}$ under $\left.\mathbb{P},\right)$ the quenched invariance principle for NNBRWs was established by Berger and Deuschel [5] under the so-called "genuinely $d$-dimensional" 
condition, where the environment is allowed to be degenerate. More recently, under such "genuinely d-dimensional" condition, Berger, Cohen, Deuschel and Guo [4] obtained some quantitative estimates for the solution to elliptic equations, as well as the elliptic Harnack inequality. For the NNBRW in timedependent balanced environments, the quenched invariance principle has been proven by Deuschel, Guo and Ramirez in [9], while the quenched local central limit theorem was established by Deuschel and Guo in $[8]$.

The goal of this paper is to establish the quenched invariance principle for long range random walks in balanced random environments, i.e., $\kappa(x, z)(\omega)$ does not vanish when $|z|>1$. The following assumption will be in force in the paper.

Assumption (A0) $\left\{\kappa(x, z): x \in \mathbb{Z}^{d}, z \in \mathbb{Z}_{+, *}^{d}\right\}$ is a sequence of independent non-negative random variables, where

$$
\mathbb{Z}_{+, *}^{d}:=\left\{x=\left(x_{1}, \cdots, x_{d}\right) \in \mathbb{Z}^{d}: x_{i_{0}}>0 \text { for } i_{0}:=\min \left\{1 \leqslant i \leqslant d: x_{i} \neq 0\right\}\right\} .
$$

Note that $\mathbb{Z}_{\mathbf{0}}^{d}=\mathbb{Z}_{+, *}^{d} \cup\left(-\mathbb{Z}_{+, *}^{d}\right)$, and $\kappa(x, z)$ satisfies the balanced condition (1.1) (i.e. $\kappa(x, z)$ is symmetric in $z \in \mathbb{Z}_{\mathbf{0}}^{d}$ for each fixed $\left.x \in \mathbb{Z}^{d}\right)$. So $\kappa(x, z)$ on $\mathbb{Z}^{d} \times \mathbb{Z}_{\mathbf{0}}^{d}$ is determined by its values on $\mathbb{Z}^{d} \times \mathbb{Z}_{+, *}^{d}$.

We will consider the following assumption when $\alpha \in(0,2)$.

Assumption (A1) Assume $\alpha \in(0,2)$ and $d>4-2 \alpha$.

(i) There is some constant $p>\max \left\{\frac{2(d+1)}{d}, \frac{d+1}{2-\alpha}\right\}$ so that

$$
\sup _{x \in \mathbb{Z}^{d}, z \in \mathbb{Z}_{0}^{d}} \mathbb{E}\left[\kappa(x, z)^{p}\right]<\infty .
$$

(ii) There exists a bounded continuous function $K: \mathbb{R}^{d} \times \mathbb{R}_{0}^{d} \rightarrow \mathbb{R}_{+}:=[0, \infty)$ such that for every large integer $R \geqslant 1$ and small $\varepsilon \in(0,1)$,

$$
\lim _{n \rightarrow \infty} \sup _{\substack{x \in \mathbb{Z}^{d}, z \in \mathbb{Z}_{0}^{d}: \\|x| \leqslant n R, \varepsilon n<|z|<n / \varepsilon}}|\mathbb{E}[\kappa(x, z)]-K(x / n, z / n)|=0,
$$

and that the solution to the martingale problem for $\left(\overline{\mathcal{L}}, C_{c}^{2}\left(\mathbb{R}^{d}\right)\right)$ is unique, where for $f \in C_{c}^{\infty}\left(\mathbb{R}^{d}\right)$

$$
\begin{aligned}
\overline{\mathcal{L}} f(x): & =\text { p.v. } \int_{\mathbb{R}_{\mathbf{0}}^{d}}(f(x+z)-f(x)) \frac{K(x, z)}{|z|^{d+\alpha}} d z \\
& =\int_{\mathbb{R}_{\mathbf{0}}^{d}}\left(f(x+z)-f(x)-\nabla f(x) \cdot z \mathbb{1}_{\{|z| \leqslant 1\}}\right) \frac{K(x, z)}{|z|^{d+\alpha}} d z,
\end{aligned}
$$

$\mathbb{R}_{\mathbf{0}}^{d}:=\mathbb{R}^{d} \backslash\{\mathbf{0}\}$, and p.v. stands for principal value.

Remark 1.1. (i) Condition (1.3) along with the continuity of $K(x, z)$ on $\mathbb{R}^{d} \times \mathbb{R}_{\mathbf{0}}^{d}$ implies that

$$
K(\lambda x, \lambda z)=K(x, z) \text { for every } x, z \in \mathbb{R}^{d} \text { and } \lambda>0,
$$

and if we write $z=r \theta \in \mathbb{R}_{\mathbf{0}}^{d}$ in spherical coordinates with $r=|z|$ and $\theta=z /|z| \in \mathbb{S}^{d-1}$, then for every $x \in \mathbb{R}^{d}$,

$$
\lim _{r \rightarrow \infty} K(x, r \theta)=\lim _{r \rightarrow \infty} K(x / r, \theta)=K(0, \theta) .
$$

In particular, $(1.5)$ says that $K(x, z)$ is uniquely determined by its value on the unit sphere in $\mathbb{R}^{2 d}$ and hence $K(x, z)$ is bounded on $\mathbb{R}^{d} \times \mathbb{R}^{d}$. 
(ii) Observe that conditions (1.1) and (1.3) along with the continuity of $K(x, z)$ on $\mathbb{R}^{d} \times \mathbb{R}_{\mathbf{0}}^{d}$ imply that $K(x, z)$ is balanced in the sense that

$$
K(x, z)=K(x,-z) \quad \text { for all } x \in \mathbb{R}^{d}, z \in \mathbb{R}_{\mathbf{0}}^{d} .
$$

Moreover, by (1.2), (1.3) and the continuity of $K(x, z)$ on $\mathbb{R}^{d} \times \mathbb{R}_{\mathbf{0}}^{d}$,

$$
\sup _{x \in \mathbb{R}^{d}, z \in \mathbb{R}_{\mathbf{0}}^{d}} K(x, z) \leqslant \sup _{x \in \mathbb{Z}^{d}, z \in \mathbb{Z}_{\mathbf{0}}^{d}} \mathbb{E} \kappa(x, z) .
$$

Note also that here we only assume the uniqueness of the martingale problem for $\left(\overline{\mathcal{L}}, C_{c}^{2}\left(\mathbb{R}^{d}\right)\right)$, since the boundedness and the continuity of $K(x, z)$ on $\mathbb{R}^{d} \times \mathbb{R}_{0}^{d}$ ensure that the existence of the martingale problem for $\left(\overline{\mathcal{L}}, C_{c}^{2}\left(\mathbb{R}^{d}\right)\right)$; see [2, Theorem 4.1]. Some sufficient conditions for the uniqueness of the solution to the martingale problem for $\mathcal{L}$ defined by (1.4) are known; for example, when $K(\cdot, \cdot)$ satisfies $0<K_{1} \leqslant K(x, z) \leqslant K_{2}<\infty$ for all $x \in \mathbb{R}^{d}$ and $z \in \mathbb{R}_{\mathbf{0}}^{d}$, and $\int_{0}^{1} r^{-1} \psi_{K}(r) d r<\infty$, where

$$
\psi_{K}(r)=\sup _{z \in \mathbb{R}_{0}^{d}, x, y \in \mathbb{R}^{d} \text { with }|x-y| \leqslant r}|K(x, z)-K(y, z)|,
$$

see [2, Theorem 1.2], [7, Theorem 4.6] and [14, Theorem 1.3] for related work.

We need the following assumption instead of Assumption (A1) when $\alpha=2$.

Assumption (A2) Assume $\alpha=2$ and $d \geqslant 1$.

(i) There are some constants $c_{*}>0$ and $\eta \in(1,2)$ such that

$$
\sup _{x \in \mathbb{Z}^{d}, z \in \mathbb{Z}_{\mathbf{0}}^{d}} \mathbb{E}\left[\exp \left(c_{*} \kappa(x, z)^{\eta}\right)\right]<\infty .
$$

(ii) There is a constant matrix $A:=\left(a_{i j}\right)_{1 \leqslant i, j \leqslant d}$ so that for every $R>1$ and $1 \leqslant i, j \leqslant d$,

$$
\lim _{n \rightarrow \infty} \sup _{x \in n^{-1} \mathbb{Z}^{d}:|x| \leqslant R}\left|\frac{1}{\log (1+n)} \sum_{z \in \mathbb{Z}_{\mathbf{0}}^{d}:|z| \leqslant n} \frac{z_{i} z_{j} \mathbb{E}[\kappa(n x, z)]}{|z|^{d+2}}-a_{i j}\right|=0 .
$$

For every $n \geqslant 1$ and $t>0$, let

$$
X_{t}^{\omega, n}:= \begin{cases}n^{-1} X_{n^{\alpha} t}^{\omega}, & \alpha \in(0,2), \\ n^{-1} X_{n^{2} t / \log (n+1)}^{\omega}, & \alpha=2 .\end{cases}
$$

For any $T>0$, denote by $\mathbb{P}_{x}^{\omega}$ (for simplicity we omit $T$ in the notation) the distribution of $X^{\omega}:=$ $\left(X_{t}^{\omega}\right)_{0 \leqslant t \leqslant T}$ on the Skorohod space $\mathscr{D}\left([0, T] ; \mathbb{R}^{d}\right)$ with initial starting point $x \in \mathbb{Z}^{d}$, and denote by $\mathbb{P}_{x}^{\omega, n}$ the distribution of $X^{\omega, n}:=\left(X_{t}^{\omega, n}\right)_{0 \leqslant t \leqslant T}$ on $\mathscr{D}\left([0, T] ; \mathbb{R}^{d}\right)$ starting at $x \in n^{-1} \mathbb{Z}^{d}$.

Theorem 1.2. (i) Suppose that $\alpha \in(0,2)$, and that Assumptions (A0) and (A1) hold. Then for every $T>0$ and a.s. $\omega \in \Omega, \mathbb{P}_{\mathbf{0}}^{\omega, n}$ converges weakly to $\overline{\mathbb{P}}_{\mathbf{0}}$ on $\mathscr{D}\left([0, T] ; \mathbb{R}^{d}\right)$, where $\overline{\mathbb{P}}_{\mathbf{0}}$ is the distribution of the unique solution to the martingale problem for $\left(\overline{\mathcal{L}}, C_{c}^{2}\left(\mathbb{R}^{d}\right)\right)$ defined by (1.4) starting at $\mathbf{0 .}$

(ii) Suppose that $\alpha=2$, and that Assumptions (A0) and (A2) hold. Then for every $T>0$ and a.s. $\omega \in \Omega, \mathbb{P}_{\mathbf{0}}^{\omega, n}$ converges weakly to $\overline{\mathbb{P}}_{\mathbf{0}}$ on $\mathscr{D}\left([0, T] ; \mathbb{R}^{d}\right)$, where $\overline{\mathbb{P}}_{\mathbf{0}}$ is the distribution of Brownian motion starting at $\mathbf{0}$ with a deterministic non-negative definite covariance matrix $A$.

We have the following sufficient condition for (1.8) in Assumption (A2)(ii).

Proposition 1.3. Suppose that $\alpha=2, d \geqslant 1$ and $\sup _{x \in \mathbb{Z}^{d}, z \in \mathbb{Z}_{0}^{d}} \mathbb{E}[\kappa(x, z)]<\infty$. If there exists a bounded and continuous function $K: \mathbb{R}_{\mathbf{0}}^{d} \rightarrow \mathbb{R}_{+}$such that for every integer $R \geqslant 1$ and $\varepsilon \in(0,1)$,

$$
\lim _{n \rightarrow \infty} \sup _{\substack{x \in n^{-1} \mathbb{Z}^{d}, z \in n^{-1} \mathbb{Z}_{0}^{d} \\|x| \leqslant n R, n^{\varepsilon} \leqslant|z| \leqslant n}}|\mathbb{E}[\kappa(x, z)]-K(z / n)|=0,
$$


then (1.8) holds with

$$
a_{i j}=\int_{\mathbb{S}^{d-1}} K(\theta) \theta_{i} \theta_{j} d \theta
$$

where $\mathbb{S}^{d-1}:=\left\{z \in \mathbb{R}^{d}:|z|=1\right\}$ and $d \theta$ is the standard Lebesgue surface measure on $\mathbb{S}^{d-1}$.

Remark 1.4. (i) Just as that in Remark 1.1, condition (1.9) together with the continuity of the function $K(z)$ on $\mathbb{R}_{\mathbf{0}}^{d}$ implies that $K(z)$ is a homogenous function of degree 0 ; that is,

$$
K(z)=K(z /|z|) \quad \text { for every } z \in \mathbb{R}_{\mathbf{0}}^{d} .
$$

(ii) To the best of our knowledge, all the existing literature including references $[4,5,8,9,11,12]$ quoted above are concerned with nearest neighbor random walks in balanced random environments. This is the first paper to investigate the quenched invariance principle for long range random walks in balanced random environments. The reader is referred to [6] for the corresponding work on random conductance models with (symmetric) $\alpha$-stable-like jumps. Compared with assumptions in $[4,5,8,9,11,12]$, our method can be applied to non-ergodic environments since $\left\{\kappa(x, z)(\omega): x \in \mathbb{Z}^{d}, z \in \mathbb{Z}_{+, *}^{d}, \omega \in \Omega\right\}$ are not required to be identically distributed.

(iii) The essential character of long range random walk in the present paper is that, the probability of jump from $x$ to $y$ is of the order $|x-y|^{-d-\alpha}$ with some $\alpha \in(0,2]$. In particular, this indicates that the second moment of the process $X$ is infinite. Therefore, we can not expect the scaling process to be a Brownian motion, or can not take the diffusive scaling to study the invariance principle. Note that, when $\alpha \in(0,2),|x-y|^{-d-\alpha}$ is a typical transition density for $\alpha$-stable random walk on $\mathbb{Z}^{d}$. So, in this case it is natural to adopt the $\alpha$-stable scaling and expect the limit process to be a stable-like process. Also due to this observation, the average of the sum for $\kappa(x, z)$ in large scale, which converges to $\mathbb{E}[\kappa(x, z)]$ by the Borel-Cantelli arguments under the independent property of $\kappa(x, z)$ and some moments conditions on $\kappa(x, z)$, will be directly involved into the jumping kernel of limit process. The case that $\alpha=2$ is distinct from $\alpha \in(0,2)$. However, by the similar method for $\alpha \in(0,2)$, it holds that with proper scaling (which is not the diffusive scaling) the limit process is a Brownian motion, whose coefficients are determined by $\mathbb{E}[\kappa(x, z)]$ as well. We should emphasize that the framework for $\alpha>2$ is completely different. Roughly speaking, when $\alpha>2$ the second moment of the process $X$ is finite. Even it is believed that by taking the diffusion scaling the limit process should be a Brownian motion, we do not know how to prove it in general. See Subsection 4.2 for more remarks.

(iv) In order to establish the quenched invariance principle and related results for NNBRWs in either ergodic environments or i.i.d. environments, some analytic tools, in particular the socalled Aleksandrov-Bakelman-Pucci (ABP) type estimates, play a crucial role; see [4, 5, 8, 9, 11, 12]. However, for the non-symmetric $\alpha$-stable-like operator $\overline{\mathcal{L}}$ defined by (1.4), the ABP type estimates are still unknown except for a very special class of coefficients $K(x, z)$, see [10] for more details. In this paper, we use a probabilistic approach to tackle the quenched invariant principle. This approach is completely different from those in the above mentioned papers. We believe that our paper provides another reasonable approach to study quenched invariance principle for random walks in balanced random environments. In particular, our approach can efficiently handle the $\alpha=2$ case, which is of interest in its own.

(v) We emphasize that, unlike random conductance models as considered in [6, Theorem 1.1], in this paper we do not require the inverse moment condition such as $\sup _{x \in \mathbb{Z}^{d}, z \in \mathbb{Z}_{0}^{d}} \mathbb{E}\left[\kappa(x, z)^{-q}\right]<\infty$ for some $q>0$. Some kind of non-degenerate condition is partly implied by the uniqueness assumption of the martingale problem for $\left(\overline{\mathcal{L}}, C_{c}^{2}\left(\mathbb{R}^{d}\right)\right)$ of $(1.4)$. For instance, all the literature we know concerning the uniqueness of the martingale problem for the non-local operator $\overline{\mathcal{L}}$ defined by (1.4) are proved under some kind of non-degeneracy of the associated coefficients. In particular, the uniqueness of the martingale problem for the operator $\overline{\mathcal{L}}$ defined by (1.4) along with (1.3) could indicate non-degeneracy of the function $x \mapsto \lim _{z \in \mathbb{Z}_{0}^{d} \text { with }|z| \rightarrow \infty} \mathbb{E}[\kappa(x, z)]$. Moreover, we do not need to assume the balanced condition (1.1) for $0<\alpha<1$; see Subsection 2.2 below. 
(vi) By (1.3) and the continuity of $K(x, z)$, it is easy to verify that $K(x, z)=K(s x, s z)$ for every $x \in \mathbb{R}^{d}, z \in \mathbb{R}_{0}^{d}$ and $s>0$, which imply that the process $\left(Y_{t}\right)_{t \geqslant 0}$ whose distribution is the unique solution to the martingale problem for $\left(\overline{\mathcal{L}}, C_{c}^{2}\left(\mathbb{R}^{d}\right)\right)$ on $\mathscr{D}\left([0, \infty) ; \mathbb{R}^{d}\right)$ as in Theorem 1.2 (i) satisfies the following scaling property

$$
\left(\lambda Y_{\lambda^{-\alpha} t}\right)_{t \geqslant 0} \stackrel{d}{=}\left(Y_{t}\right)_{t \geqslant 0}, \quad \lambda>0 .
$$

Here $\stackrel{d}{=}$ means the distribution of two processes are the same. Moreover, the limiting operator $\overline{\mathcal{L}}$ defined by (1.4) may not be symmetric with respect to the Lebesgue measure, which is different from the behavior of NNBRWs studied in $[4,5,8,9,11,12]$. This non-symmetry nature of the limit operator $\overline{\mathcal{L}}$ is due to the fact that $\left\{\kappa(x, z)(\omega): x \in \mathbb{Z}^{d}, z \in \mathbb{Z}_{+, *}^{d}, \omega \in \Omega\right\}$ may not be identically distributed.

(vii) By the proof below, we see that the conclusion of Theorem 1.2 (ii) holds true if the condition (1.8) is replaced by the following

$$
\lim _{n \rightarrow \infty} \sup _{x \in n^{-1} \mathbb{Z}^{d}:|x| \leqslant R}\left|\frac{1}{\log (1+n)} \sum_{z \in \mathbb{Z}_{0}^{d}:|z| \leqslant n} \frac{z_{i} z_{j} \mathbb{E}[\kappa(n x, z)]}{|z|^{d+2}}-a_{i j}(x)\right|=0
$$

for all integer $R \geqslant 1$ and $1 \leqslant i, j \leqslant d$, where $a_{i j}(x): \mathbb{R}^{d} \rightarrow \mathbb{R}$ is a bounded and continuous function. It further follows from (1.10) and the continuity of $a_{i j}$ that

$$
a_{i j}(s x)=a_{i j}(x) \text { for every } x \in \mathbb{R}^{d} \text { and } s>0 .
$$

In particular, for any $x \in \mathbb{R}^{d}, a_{i j}(x)=\lim _{s \rightarrow 0} a_{i j}(s x)=a_{i j}(\mathbf{0})$; that is, $a_{i, j}(x)$ is a constant function. Hence, (1.10) is essentially equivalent to (1.8).

(viii) Note that by (1.8), for every $\xi=\left(\xi_{1}, \cdots, \xi_{d}\right) \in \mathbb{R}^{d}$ and $x \in \mathbb{Z}^{d}$, it holds that

$$
\begin{aligned}
\sum_{i, j=1}^{d} a_{i j} \xi_{i} \xi_{j} & =\lim _{n \rightarrow \infty} \frac{1}{\log (1+n)} \sum_{z \in \mathbb{Z}_{0}^{d}:|z| \leqslant n} \sum_{i, j=1}^{d} \frac{\xi_{i} \xi_{j} z_{i} z_{j} \mathbb{E}[\kappa(x, z)]}{|z|^{d+2}} \\
& =\lim _{n \rightarrow \infty} \frac{1}{\log (1+n)} \sum_{z \in \mathbb{Z}_{\mathbf{0}}^{d}:|z| \leqslant n}\left(\sum_{i=1}^{d} \xi_{i} z_{i}\right)^{2} \frac{\mathbb{E}[\kappa(x, z)]}{|z|^{d+2}} \geqslant 0 .
\end{aligned}
$$

This immediately implies that $A=\left(a_{i j}\right)_{1 \leqslant i, j \leqslant d}$ is non-negative definite. Furthermore, by (1.8) and (1.11), it is not difficult to verify that if $\liminf _{|z| \rightarrow \infty} \mathbb{E}[\kappa(x, z)]>0$ for every $x \in \mathbb{Z}^{d}$, then $\sum_{i, j=1}^{d} a_{i j} \xi_{i} \xi_{j}>0$ for every non-zero $\xi \in \mathbb{R}^{d}$, and so $A$ is non-degenerate. Similarly, if the function $K: \mathbb{R}_{\mathbf{0}}^{d} \rightarrow \mathbb{R}_{+}$in Proposition 1.3 satisfies that $K\left(\theta_{0}\right)>0$ for some $\theta_{0} \in \mathbb{S}^{d-1}$, then $A$ is also non-degenerate. Indeed, by the continuity of $K$, there exists an open neighborhood of $U \subset \mathbb{S}^{d-1}$ containing $\theta_{0}$ such that $\inf _{\theta \in U} K(\theta)>0$. On the other hand, for any non-zero $\xi \in \mathbb{R}^{d}$, we can find an open subset $U_{0} \subset U$ satisfying that $\inf _{\theta \in U_{0}}|\langle\xi, \theta\rangle|>0$, where $\langle\xi, \theta\rangle:=\sum_{i=1}^{d} \xi_{i} \theta_{i}$. Therefore, by Proposition 1.3,

$$
\sum_{i, j=1}^{d} a_{i j} \xi_{i} \xi_{j}=\int_{\mathbb{S}^{d-1}} K(\theta)|\langle\xi, \theta\rangle|^{2} d \theta \geqslant \int_{U_{0}} K(\theta)|\langle\xi, \theta\rangle|^{2} d \theta \geqslant \inf _{\theta \in U_{0}}\left(K(\theta)|\langle\xi, \theta\rangle|^{2}\right) \int_{U_{0}} d \theta>0 .
$$

The rest of the paper is organized as follows. In the next section, we consider a deterministic environment and establish the invariance principle for long range balanced random walks. The main result for $\alpha \in(0,2)$ is Theorem 2.4. Our approach is based on the tail probability estimates for the exit time of stable-like balanced random walks and the martingale method. When $\alpha \in(0,1)$, we can get rid of the balanced condition (1.1) with some slight modifications of the proof of Theorem 2.4; see Theorem 2.6. When $\alpha=2$, the main idea of the proof for Theorem 2.4 still works; see Theorem 2.7. Section 3 is devoted to the proofs of Theorem 1.2 and Proposition 1.3. Some extensions and remarks of our main results are given in the last section. 


\section{INVARIANCE PRINCIPLE FOR LONG RANGE BALANCED RANDOM WALKS}

In this section, we fix the environment $\omega$ in $\left\{\kappa(x, z)(\omega): x, z \in \mathbb{Z}^{d}\right\}$; in other words, we consider a deterministic environment and discuss the invariance principle for its corresponding purely discontinuous Markov process on $\mathbb{Z}^{d}$.

Let $X:=\left(X_{t}\right)_{t \geqslant 0}$ be a Markov process on $\mathbb{Z}^{d}$ associated with the following infinitesimal generator

$$
\mathcal{L} f(x):=\sum_{z \in \mathbb{Z}_{0}^{d}}(f(x+z)-f(x)) \frac{\kappa(x, z)}{|z|^{d+\alpha}}, \quad f \in B_{b}\left(\mathbb{Z}^{d}\right),
$$

where $\alpha \in(0,2]$ and $\kappa(\cdot, \cdot): \mathbb{Z}^{d} \times \mathbb{Z}_{0}^{d} \rightarrow[0, \infty)$. Denote by $\mathbb{P}_{x}$ the distribution of $X$ on $\mathscr{D}\left([0, \infty) ; \mathbb{Z}^{d}\right)$ endowed with the Skorohod topology and with initial point $x \in \mathbb{Z}^{d}$.

For any $\alpha \in(0,2]$ and $n \geqslant 1$, consider the scaled process

$$
X^{n}:=\left\{X_{t}^{n}: t \geqslant 0\right\}:= \begin{cases}\left\{n^{-1} X_{n^{\alpha} t}: t \geqslant 0\right\}, & \alpha \in(0,2), \\ \left\{n^{-1} X_{n^{2} t / \log (1+n)}: t \geqslant 0\right\}, & \alpha=2,\end{cases}
$$

which takes values in $n^{-1} \mathbb{Z}^{d}$. Clearly $X^{n}$ is a strong Markov process on $n^{-1} \mathbb{Z}^{d}$, and it is easy to check that it has the corresponding infinitesimal generator

$$
\mathcal{L}_{n} f(x):= \begin{cases}n^{-d} \sum_{z \in n^{-1} \mathbb{Z}_{\mathbf{0}}^{d}}(f(x+z)-f(x)) \frac{\kappa(n x, n z)}{|z|^{d+\alpha}}, & \alpha \in(0,2), \\ \left(n^{d} \log (1+n)\right)^{-1} \sum_{z \in n^{-1} \mathbb{Z}_{\mathbf{0}}^{d}}(f(x+z)-f(x)) \frac{\kappa(n x, n z)}{|z|^{d+2}}, & \alpha=2\end{cases}
$$

acting on $f \in B_{b}\left(n^{-1} \mathbb{Z}^{d}\right)$. Denote by $\mathbb{P}_{x}^{n}$ the distribution of $X^{n}$ on $\mathscr{D}\left([0, \infty) ; n^{-1} \mathbb{Z}^{d}\right)$ starting at $x \in$ $n^{-1} \mathbb{Z}^{d}$

2.1. Balanced case for $\alpha \in(0,2)$. Throughout this subsection, we assume $\alpha \in(0,2)$ and the balanced condition (1.1).

Assumption (B1). There exist constants $\theta \in(0,1), C_{1}>0$ and $R_{0} \geqslant 1$ such that for every $R>R_{0}$ and $r \in\left[R^{\theta}, R\right]$,

$$
\sup _{x \in B(\mathbf{0}, 2 R)} \sum_{z \in \mathbb{Z}^{d}: 1 \leqslant|z| \leqslant r} \frac{\kappa(x, z)}{|z|^{d+\alpha-2}} \leqslant C_{1} r^{2-\alpha}
$$

and

$$
\sup _{x \in B(\mathbf{0}, 2 R)} \sum_{z \in \mathbb{Z}^{d}:|z|>r} \frac{\kappa(x, z)}{|z|^{d+\alpha}} \leqslant C_{1} r^{-\alpha}
$$

Lemma 2.1. Suppose that Assumption (B1) holds. Then, we have

(i) There is a constant $c>0$ that depends only on the constant $C_{1}$ in Assumption (B1) so that for every $R>R_{0}, r \in\left[R^{\theta}, R\right], x \in B(\mathbf{0}, R)$ and $t>0$,

$$
\mathbb{P}_{x}\left(\tau_{B(x, r)} \leqslant t\right) \leqslant c t / r^{\alpha} .
$$

Here and in what follows, for any subset $A \subset \mathbb{Z}^{d}, \tau_{A}=\inf \left\{t>0: X_{t} \notin A\right\}$ is the first exit time from $A$ by the process $X$.

(ii) $\left\{\mathbb{P}_{0}^{n}\right\}_{n=1}^{\infty}$ is tight in $\mathscr{D}\left([0, T] ; \mathbb{R}^{d}\right)$ for any $T>0$.

Proof. (i) For any $r>0$ and $x \in \mathbb{R}^{d}$, take $f_{x, r} \in C_{b}^{2}\left(\mathbb{R}^{d}\right)$ such that

$$
f_{x, r}(z)= \begin{cases}0, & 0 \leqslant|z-x| \leqslant r / 2 \\ \in[0,1], & r / 2<|z-x|<r \\ 1, & |z-x| \geqslant r\end{cases}
$$


$\sup _{z \in \mathbb{R}^{d}}\left|\nabla f_{x, r}(z)\right| \leqslant c_{0} r^{-1}$, and $\sup _{z \in \mathbb{R}^{d}}\left|\nabla^{2} f_{x, r}(z)\right| \leqslant c_{0} r^{-2}$ for some constant $c_{0}>0$ independent of $r$ and $x$. Then, for every $x \in B(\mathbf{0}, R)$,

$$
\mathbb{P}_{x}\left(\tau_{B(x, r)} \leqslant t\right) \leqslant \mathbb{E}_{x} f_{x, r}\left(X_{\left.t \wedge \tau_{B(x, r)}\right)}\right)=\mathbb{E}_{x}\left[\int_{0}^{t \wedge \tau_{B(x, r)}} \mathcal{L} f_{x, r}\left(X_{s}\right) d s\right]
$$

Let $R_{0} \geqslant 1$ be the constant in Assumption (B1). For every $R>R_{0}, r \in\left[R^{\theta}, R\right], x \in B(\mathbf{0}, R)$ and $y \in B(x, r)$,

$$
\begin{aligned}
\mathcal{L} f_{x, r}(y)= & \sum_{z \in \mathbb{Z}^{d: 1 \leqslant|z| \leqslant r}}\left(f_{x, r}(y+z)-f_{x, r}(y)-\nabla f_{x, r}(y) \cdot z\right) \frac{\kappa(y, z)}{|z|^{d+\alpha}} \\
& +\sum_{z \in \mathbb{Z}^{d}:|z| \geqslant r}\left(f_{x, r}(y+z)-f_{x, r}(y)\right) \frac{\kappa(y, z)}{|z|^{d+\alpha}} \\
= & : I_{1, r}+I_{2, r},
\end{aligned}
$$

where we used (1.1) in the first equality.

According to (2.2), for every $R>R_{0}, r \in\left[R^{\theta}, R\right], x \in B(\mathbf{0}, R)$ and $y \in B(x, r)$,

$$
\left|I_{1, r}\right| \leqslant \frac{1}{2}\left\|\nabla^{2} f_{x, r}\right\|_{\infty} \sum_{z \in \mathbb{Z}^{d}: 1 \leqslant|z| \leqslant r} \frac{\kappa(y, z)}{|z|^{d+\alpha-2}} \leqslant \frac{c_{0}}{2} r^{-2} \sup _{y \in B(\mathbf{0}, 2 R)} \sum_{z \in \mathbb{Z}^{d}: 1 \leqslant|z| \leqslant r} \frac{\kappa(y, z)}{|z|^{d+\alpha-2}} \leqslant \frac{c_{0} C_{1}}{2} r^{-\alpha} .
$$

Similarly, we have by (2.3),

$$
\sup _{x \in B(\mathbf{0}, R), y \in B(x, r)}\left|I_{2, r}\right| \leqslant 2 C_{1} r^{-\alpha} .
$$

Hence, for every $R>R_{0}$ and $r \in\left[R^{\theta}, R\right]$,

$$
\sup _{x \in B(\mathbf{0}, R), y \in B(x, r)}\left|\mathcal{L} f_{x, r}(y)\right| \leqslant 2\left(1+c_{0} / 4\right) C_{1} r^{-\alpha} .
$$

Combining all the estimates above, we obtain (2.4).

(ii) For a Borel subset $A \subset n^{-1} \mathbb{Z}^{d}$, let $\tau_{A}^{n}:=\inf \left\{t>0: X_{t}^{n} \notin A\right\}$ be the first exit time from $A$ by the process $X^{n}$. By the fact that $X_{t}^{n}=n^{-1} X_{n^{\alpha} t}$ and (2.4), for every fixed integer $R \geqslant 1$ and $n>R_{0} / R$, we have

$$
\mathbb{P}_{\mathbf{0}}^{n}\left(\sup _{t \in[0, T]}\left|X_{t}^{n}\right|>R\right) \leqslant \mathbb{P}_{\mathbf{0}}^{n}\left(\tau_{B(\mathbf{0}, R)}^{n} \leqslant T\right)=\mathbb{P}_{\mathbf{0}}\left(\tau_{B(\mathbf{0}, n R)} \leqslant n^{\alpha} T\right) \leqslant c n^{\alpha} T(n R)^{-\alpha}=c T / R^{\alpha} .
$$

Consequently,

$$
\lim _{R \rightarrow \infty} \limsup _{n \rightarrow \infty} \mathbb{P}_{\mathbf{0}}^{n}\left(\sup _{t \in[0, T]}\left|X_{t}^{n}\right|>R\right)=0 .
$$

On the other hand, for any $\eta>0$, any sequence of stopping times $\left\{\tau_{n}\right\}_{n \geqslant 1}$ of $\left\{X^{n}\right\}_{n \geqslant 1}$ such that $\tau_{n} \leqslant T$, and any sequence $\left\{\varepsilon_{n}\right\}_{n \geqslant 1}$ such that $\lim _{n \rightarrow \infty} \varepsilon_{n}=0$, it follows from the strong Markov property of $X^{n}$ that

$$
\begin{aligned}
\mathbb{P}_{\mathbf{0}}^{n}\left(\left|X_{\tau_{n}+\varepsilon_{n}}^{n}-X_{\tau_{n}}^{n}\right|>\eta\right) & =\mathbb{E}_{\mathbf{0}}^{n}\left[\mathbb{P}_{X_{\tau_{n}}^{n}}^{n}\left(\left|X_{\varepsilon_{n}}^{n}-X_{0}^{n}\right|>\eta\right)\right] \\
& \leqslant \mathbb{P}_{\mathbf{0}}^{n}\left(\tau_{B(\mathbf{0}, R)}^{n} \leqslant T\right)+\sup _{x \in B(\mathbf{0}, R)} \mathbb{P}_{x}^{n}\left(\tau_{B(x, \eta)}^{n} \leqslant \varepsilon_{n}\right) \\
& =\mathbb{P}_{\mathbf{0}}\left(\tau_{B(\mathbf{0}, n R)} \leqslant n^{\alpha} T\right)+\sup _{x \in B(\mathbf{0}, n R)} \mathbb{P}_{x}\left(\tau_{B(\mathbf{0}, n \eta)} \leqslant n^{\alpha} \varepsilon_{n}\right),
\end{aligned}
$$

where in the first inequality we used the fact $\tau_{n} \leqslant T$ for the second term. Taking $n$ large enough so that $n R>R_{0}$ and $n \eta>(n R)^{\theta}$, we get from (2.4) that

$$
\limsup _{n \rightarrow \infty} \mathbb{P}_{\mathbf{0}}^{n}\left(\left|X_{\tau_{n}+\varepsilon_{n}}^{n}-X_{\tau_{n}}^{n}\right|>\eta\right) \leqslant c \limsup _{n \rightarrow \infty}\left(\frac{n^{\alpha} T}{(n R)^{\alpha}}+\frac{n^{\alpha} \varepsilon_{n}}{(n \eta)^{\alpha}}\right) \leqslant c T / R^{\alpha},
$$


which, by taking $R \rightarrow \infty$, yields

$$
\limsup _{n \rightarrow \infty} \mathbb{P}_{\mathbf{0}}^{n}\left(\left|X_{\tau_{n}+\varepsilon_{n}}^{n}-X_{\tau_{n}}^{n}\right|>\eta\right)=0 .
$$

The desired tightness assertion now follows from (2.7), (2.8) and [1, Theorem 1].

Lemma 2.2. Under Assumption (B1), for every $f \in C_{c}^{2}\left(\mathbb{R}^{d}\right)$,

$$
\sup _{n \geqslant 1} \sup _{x \in n^{-1} \mathbb{Z}^{d}}\left|\mathcal{L}_{n} f(x)\right|<\infty
$$

and

$$
\lim _{R \rightarrow \infty} \limsup _{n \rightarrow \infty} \sup _{x \in n^{-1} \mathbb{Z}^{d}:|x| \geqslant R}\left|\mathcal{L}_{n} f(x)\right|=0 .
$$

Proof. Fix $f \in C_{c}^{2}\left(\mathbb{R}^{d}\right)$. Suppose that $\operatorname{supp}(f) \subset B\left(\mathbf{0}, N_{0}\right)$ for some $N_{0} \geqslant 1$. Then for every $x \in$ $B\left(\mathbf{0}, 4 N_{0}\right) \cap n^{-1} \mathbb{Z}^{d}$, we have by (2.1) that for $n$ large enough,

$$
\begin{aligned}
\left|\mathcal{L}_{n} f(x)\right| \leqslant & n^{-d} \sum_{z \in n^{-1} \mathbb{Z}_{0}^{d}:|z| \leqslant 1}|f(x+z)-f(x)-\nabla f(x) \cdot z| \frac{\kappa(n x, n z)}{|z|^{d+\alpha}} \\
& +n^{-d} \sum_{z \in n^{-1} \mathbb{Z}^{d}:|z|>1}|f(x+z)-f(x)| \frac{\kappa(n x, n z)}{|z|^{d+\alpha}} \\
\leqslant & \frac{1}{2} n^{\alpha-2}\left\|\nabla^{2} f\right\|_{\infty} \sup _{x \in B\left(\mathbf{0}, 4 n N_{0}\right)} \sum_{z \in \mathbb{Z}_{0}^{d}:|z| \leqslant n} \frac{\kappa(x, z)}{|z|^{d+\alpha-2}} \\
& +2 n^{\alpha}\|f\|_{\infty} \sup _{x \in B\left(\mathbf{0}, 4 n N_{0}\right)} \sum_{z \in \mathbb{Z}^{d}:|z|>n} \frac{\kappa(x, z)}{|z|^{d+\alpha}} \\
\leqslant & \frac{C_{1}}{2} n^{\alpha-2}\left\|\nabla^{2} f\right\|_{\infty} n^{2-\alpha}+2 C_{1} n^{\alpha}\|f\|_{\infty} n^{-\alpha}=2 C_{1}\left(\left\|\nabla^{2} f\right\|_{\infty}+\|f\|_{\infty}\right),
\end{aligned}
$$

where we used (1.1) in the first inequality, and (2.2) and (2.3) in the last inequality.

On the other hand, since $f$ is supported in $B\left(\mathbf{0}, N_{0}\right)$, if $|x|>4 N_{0}$, then $f(x+z)-f(x)=0$ when $|x+z|>N_{0}$. Hence for any $|x|>4 N_{0}$ and $n$ large enough, we have by (2.1) that

$$
\begin{aligned}
\left|\mathcal{L}_{n} f(x)\right| & \leqslant\|f\|_{\infty} n^{-d} \sum_{z \in n^{-1} \mathbb{Z}_{0}^{d}:|x+z| \leqslant N_{0}} \frac{\kappa(n x, n z)}{|z|^{d+\alpha}} \leqslant\|f\|_{\infty} n^{-d} \sum_{z \in n^{-1} \mathbb{Z}^{d}:|x| / 2 \leqslant|z| \leqslant 2|x|} \frac{\kappa(n x, n z)}{|z|^{d+\alpha}} \\
& \leqslant\|f\|_{\infty} n^{\alpha} \sum_{z \in \mathbb{Z}^{d}: n|x| / 2 \leqslant|z| \leqslant 2 n|x|} \frac{\kappa(n x, z)}{|z|^{d+\alpha}} \leqslant 4\|f\|_{\infty} n^{\alpha}|n x|^{-2} \sum_{z \in \mathbb{Z}_{0}^{d}:|z| \leqslant 2 n|x|} \frac{\kappa(n x, z)}{|z|^{d+\alpha-2}} \\
& \leqslant 4 C_{1}\|f\|_{\infty} n^{\alpha-2}|x|^{-2}(2 n|x|)^{2-\alpha}=c_{1}\|f\|_{\infty}|x|^{-\alpha},
\end{aligned}
$$

where we have used (2.2) in the last inequality. This proves (2.10), and along with (2.11) also yields $(2.9)$.

We need the following assumption for the convergence of $\left\{X^{n}\right\}_{n \geqslant 1}$.

Assumption (B2). There exists a bounded continuous function $K(x, z): \mathbb{R}^{d} \times \mathbb{R}_{\mathbf{0}}^{d} \rightarrow(0, \infty)$ such that $K(x, z)=K(x,-z)$ for all $x \in \mathbb{R}^{d}$ and $z \in \mathbb{R}_{\mathbf{0}}^{d}$, and that for any integer $R \geqslant 1$ and any $f \in C_{c}^{2}\left(\mathbb{R}^{d}\right)$,

$$
\liminf _{\varepsilon \rightarrow 0} \lim _{n \rightarrow \infty} n^{\alpha} \sup _{\substack{x \in \mathbb{Z}^{d}: \\|x| \leqslant n R}}\left|\sum_{\substack{z \in \mathbb{Z}^{d:} \\ n \varepsilon<|z|<n / \varepsilon}}\left(f\left(\frac{x+z}{n}\right)-f\left(\frac{x}{n}\right)\right)\left(\frac{\kappa(x, z)-K\left(\frac{x}{n}, \frac{z}{n}\right)}{|z|^{d+\alpha}}\right)\right|=0 .
$$

Clearly in the above assumption, the phase "for any integer $R \geqslant 1$ " can be replaced by "for any constant $R>0$ ". 
Lemma 2.3. Suppose that Assumptions (B1) and (B2) hold. Then for any $f \in C_{c}^{2}\left(\mathbb{R}^{d}\right)$,

$$
\lim _{n \rightarrow \infty} \sup _{x \in n^{-1} \mathbb{Z}^{d}}\left|\mathcal{L}_{n} f(x)-\overline{\mathcal{L}} f(x)\right|=0,
$$

where the operator $\overline{\mathcal{L}}$ is defined by (1.4).

Proof. For every $\eta \in(0,1)$, set for $x \in n^{-1} \mathbb{Z}^{d}$

$$
\begin{aligned}
\mathcal{L}_{n, \eta} f(x): & =n^{-d} \sum_{\substack{z \in n^{-1} \mathbb{Z}^{d}: \\
\eta<|z|<1 / \eta}}(f(x+z)-f(x)) \frac{\kappa(n x, n z)}{|z|^{d+\alpha}} \\
& =n^{\alpha} \sum_{z \in \mathbb{Z}^{d}: n \eta<|z|<n \eta^{-1}}\left(f\left(x+\frac{z}{n}\right)-f(x)\right) \frac{\kappa(n x, z)}{|z|^{d+\alpha}}, \\
\overline{\mathcal{L}}_{n, \eta} f(x): & =n^{-d} \sum_{\substack{z \in n^{-1} \mathbb{Z}^{d}: \\
\eta<|z|<1 / \eta}}(f(x+z)-f(x)) \frac{K(x, z)}{|z|^{d+\alpha}} \\
& =n^{\alpha} \sum_{\substack{z \in \mathbb{Z}^{d}: n \eta<|z|<n \eta^{-1} \\
\sum^{d+\alpha}}}\left(f\left(x+\frac{z}{n}\right)-f(x)\right) \frac{K(x, z / n)}{|z|^{d+\alpha}}
\end{aligned}
$$

and for $x \in \mathbb{R}^{d}$,

$$
\overline{\mathcal{L}}_{\eta} f(x):=\int_{\left\{z \in \mathbb{R}^{d}: \eta<|z|<\eta^{-1}\right\}}(f(x+z)-f(x)) \frac{K(x, z)}{|z|^{d+\alpha}} d z .
$$

For every $R>1$ and $\eta \in(0,1)$,

$$
\begin{aligned}
& \sup _{x \in n^{-1} \mathbb{Z}^{d}}\left|\mathcal{L}_{n} f(x)-\overline{\mathcal{L}} f(x)\right| \\
& \leqslant \sup _{x \in n^{-1} \mathbb{Z}^{d}:|x| \leqslant R}\left|\mathcal{L}_{n, \eta} f(x)-\overline{\mathcal{L}}_{\eta} f(x)\right|+\sup _{x \in n^{-1} \mathbb{Z}^{d}:|x|>R}\left|\mathcal{L}_{n} f(x)\right|+\sup _{x \in n^{-1} \mathbb{Z}^{d}:|x|>R}|\overline{\mathcal{L}} f(x)| \\
& \quad+n^{-d} \sup _{x \in n^{-1} \mathbb{Z}^{d}:|x| \leqslant R}\left|\sum_{z \in n^{-1} \mathbb{Z}_{0}^{d}:|z| \leqslant \eta}(f(x+z)-f(x)-\nabla f(x) \cdot z) \frac{\kappa(n x, n z)}{|z|^{d+\alpha}}\right| \\
& \quad+n^{-d} \sup _{x \in n^{-1} \mathbb{Z}^{d}:|x| \leqslant R} \mid \sum_{z \in n^{-1} \mathbb{Z}^{d}:|z| \geqslant \eta^{-1}}(f(x+z)-f(x)) \frac{\kappa(n x, n z)}{|z|^{d+\alpha} \mid} \\
& \quad+\sup _{x \in n^{-1} \mathbb{Z}^{d}:|x| \leqslant R}\left|\int_{\{0<|z| \leqslant \eta\} \cup\left\{|z| \geqslant \eta^{-1}\right\}}\left(f(x+z)-f(x)-\nabla f(x) \cdot z \mathbb{1}_{\{|z| \leqslant 1\}}\right) \frac{K(x, z)}{|z|^{d+\alpha}} d z\right| \\
& =: I_{1}^{n, R, \eta}+I_{2}^{n, R}+I_{3}^{n, R}+I_{4}^{n, R, \eta}+I_{5}^{n, R, \eta}+I_{6}^{n, R, \eta} .
\end{aligned}
$$

Note that due to balanced conditions (1.1) and (1.6), we may add the gradient term $\nabla f(x) \cdot z$ in the summation.

By (2.13), there is a sequence of positive numbers $\left\{\varepsilon_{k}\right\}_{k \geqslant 1} \subset(0,1)$ that decreases to 0 so that

$$
\lim _{k \rightarrow \infty} \lim _{n \rightarrow \infty} n^{\alpha} \sup _{\substack{x \in \mathbb{Z}^{d:} \\|x| \leqslant n R}}\left|\sum_{\substack{z \in \mathbb{Z}^{d}: \\ n \varepsilon_{k}<|z|<n / \varepsilon_{k}}}\left(f\left(\frac{x+z}{n}\right)-f\left(\frac{x}{n}\right)\right)\left(\frac{\kappa(x, z)-K\left(\frac{x}{n}, \frac{z}{n}\right)}{|z|^{d+\alpha}}\right)\right|=0 .
$$

Thus we have

$$
\liminf _{k \rightarrow \infty} \lim _{n \rightarrow \infty} \sup _{x \in n^{-1} \mathbb{Z}^{d}:|x| \leqslant R}\left|\mathcal{L}_{n, \varepsilon_{k}} f(x)-\overline{\mathcal{L}}_{n, \varepsilon_{k}} f(x)\right|=0 .
$$

Since $K(x, z)$ is uniformly continuous on $\left\{(x, z) \in \mathbb{R}^{2 d}:|x| \leqslant R\right.$ and $\left.\varepsilon_{k}<|z|<\varepsilon_{k}^{-1}\right\}$ for fixed $k \geqslant 1$, it is routine to show that for any $f \in C_{c}^{2}\left(\mathbb{R}^{d}\right)$ and $k \geqslant 1$,

$$
\lim _{n \rightarrow \infty} \sup _{x \in n^{-1} \mathbb{Z}^{d}:|x| \leqslant R}\left|\overline{\mathcal{L}}_{n, \varepsilon_{k}} f(x)-\overline{\mathcal{L}}_{\varepsilon_{k}} f(x)\right|=0 .
$$


Hence, for any $R>1$,

$$
\liminf _{k \rightarrow \infty} \lim _{n \rightarrow \infty} I_{1}^{n, R, \varepsilon_{k}}=0 .
$$

On the other hand, by (2.10),

$$
\lim _{R \rightarrow \infty} \limsup _{n \rightarrow \infty} I_{2}^{n, R}=0 .
$$

Following the proof for (2.9), and applying (2.2) and (2.3) respectively, we can get

$$
\limsup _{n \rightarrow \infty} I_{4}^{n, R, \eta} \leqslant c_{1} \eta^{2-\alpha}, \text { and } \limsup _{n \rightarrow \infty} I_{5}^{n, R, \eta} \leqslant c_{1} \eta^{\alpha} .
$$

Since $K$ is bounded, it is obvious that

$$
\lim _{R \rightarrow \infty} \limsup _{n \rightarrow \infty} I_{3}^{n, R}=0, \text { and } \limsup _{n \rightarrow \infty} I_{6}^{n, R, \eta} \leqslant c_{2}\left(\eta^{2-\alpha}+\eta^{\alpha}\right) .
$$

Now, we take $\eta=\varepsilon_{k}$ in the estimate (2.16). Combining all estimates above with (2.16), first letting $n \rightarrow \infty$, then taking $R \rightarrow \infty$ and $k \rightarrow \infty$, we obtain (2.14).

Now, we can state the main result in this subsection.

Theorem 2.4. Suppose that Assumptions (B1) and (B2) hold, and the solution of the martingale problem for $\left(\overline{\mathcal{L}}, C_{c}^{2}\left(\mathbb{R}^{d}\right)\right)$ defined by $(1.4)$ is unique. Then, for every $T>0, \mathbb{P}_{\mathbf{0}}^{n}$ converges weakly to $\overline{\mathbb{P}}_{\mathbf{0}}$, where $\overline{\mathbb{P}}_{\mathbf{0}}$ denotes the distribution (restricted on the time interval $[0, T]$ ) of the unique solution to the martingale problem of the operator $\left(\overline{\mathcal{L}}, C_{c}^{2}\left(\mathbb{R}^{d}\right)\right)$.

Proof. According to Lemma 2.1, $\left\{\mathbb{P}_{\mathbf{0}}^{n}\right\}_{n \geqslant 1}$ is tight. Then, there exists a weakly convergent subsequence $\left\{\mathbb{P}_{\mathbf{0}}^{n_{k}}\right\}_{k \geqslant 1}$ (which we also denote by $\left\{\mathbb{P}_{\mathbf{0}}^{n}\right\}_{n \geqslant 1}$ for simplicity) with a weak limit $\tilde{\mathbb{P}}_{\mathbf{0}}$. By our assumption, the solution of martingale problem for $\left(\overline{\mathcal{L}}, C_{c}^{2}\left(\mathbb{R}^{d}\right)\right)$ is unique. Therefore, it suffices to prove the weak limit $\tilde{\mathbb{P}}_{\mathbf{0}}$ solves the martingale problem for $\left(\overline{\mathcal{L}}, C_{c}^{2}\left(\mathbb{R}^{d}\right)\right)$ with initial value $\mathbf{0}$.

By the Skorohod theorem, we can find a probability space $(\Omega, \mathscr{F}, \hat{\mathbb{P}})$ and a family of processes $\left(X_{t}^{n}\right)_{t \geqslant 0}$ and $\left(X_{t}\right)_{t \geqslant 0}$ on this space such that

(i) The laws of $\left(X_{t}^{n}\right)_{t \geqslant 0}$ and $\left(X_{t}\right)_{t \geqslant 0}$ under $\hat{\mathbb{P}}$ are $\mathbb{P}_{\mathbf{0}}^{n}$ and $\tilde{\mathbb{P}}_{\mathbf{0}}$, respectively;

(ii) For every $T>0$, $\hat{\mathbb{P}}$-a.s. the process $X_{\text {. }}^{n}$ converges to $X$. on $\mathscr{D}\left([0, T] ; \mathbb{R}^{d}\right)$.

Since $\left(X_{t}^{n}\right)_{t \geqslant 0}$ is a solution to the martingale problem of $\left(\mathcal{L}_{n}, B_{b}\left(n^{-1} \mathbb{Z}^{d}\right)\right)$, for every $0 \leqslant s_{1} \leqslant \cdots \leqslant$ $s_{k} \leqslant s \leqslant t \leqslant T, f \in C_{c}^{2}\left(\mathbb{R}^{d}\right)$ and $G \in C_{b}\left(\left(\mathbb{R}^{d}\right)^{k}\right)$ with $k \geqslant 1$,

$$
\hat{\mathbb{E}}\left[\left(f\left(X_{t}^{n}\right)-f\left(X_{s}^{n}\right)-\int_{s}^{t} \mathcal{L}_{n} f\left(X_{r}^{n}\right) d r\right) G\left(X_{s_{1}}^{n}, \cdots X_{s_{k}}^{n}\right)\right]=0 .
$$

Note that by (2.9), the random variable inside the above expectation is uniformly bounded in $n \geqslant 1$. As $\hat{\mathbb{P}}$-a.s. the process $X^{n}$ converges to $X$. on $\mathscr{D}\left([0, T] ; \mathbb{R}^{d}\right)$, by the bounded convergence theorem,

$$
\lim _{n \rightarrow \infty} \hat{\mathbb{E}}\left[\left|G\left(X_{s_{1}}^{n}, \cdots X_{s_{k}}^{n}\right)-G\left(X_{s_{1}}, \cdots X_{s_{k}}\right)\right|\right]=0
$$

On the other hand,

$$
\begin{aligned}
& \hat{\mathbb{E}}\left[\left|\int_{s}^{t} \mathcal{L}_{n} f\left(X_{r}^{n}\right) d r-\int_{s}^{t} \overline{\mathcal{L}} f\left(X_{r}\right) d r\right|\right] \\
& \leqslant(t-s) \sup _{x \in n^{-1} \mathbb{Z}^{d}}\left|\mathcal{L}_{n} f(x)-\overline{\mathcal{L}} f(x)\right|+\hat{\mathbb{E}}\left[\left|\int_{s}^{t} \overline{\mathcal{L}} f\left(X_{r}^{n}\right) d r-\int_{s}^{t} \overline{\mathcal{L}} f\left(X_{r}\right) d r\right|\right] .
\end{aligned}
$$

We next claim that $\overline{\mathcal{L}} f \in C_{b}\left(\mathbb{R}^{d}\right)$ for any $f \in C_{c}^{2}\left(\mathbb{R}^{d}\right)$. Indeed, it follows from the boundedness of $K(x, z)$ that $\overline{\mathcal{L}} f$ is bounded in $\mathbb{R}^{d}$ for any $f \in C_{c}^{2}\left(\mathbb{R}^{d}\right)$. On the other hand, by the continuity and the boundedness of $K(x, z)$, we know $\overline{\mathcal{L}}_{\eta} f \in C_{b}\left(\mathbb{R}^{d}\right)$ for all $f \in C_{c}^{2}\left(\mathbb{R}^{d}\right)$ and $\eta>0$, where $\overline{\mathcal{L}}_{\eta} f$ is defined by (2.15). Following the proof of Lemma 2.3, we can obtain

$$
\sup _{x \in \mathbb{R}^{d}}\left|\overline{\mathcal{L}} f(x)-\overline{\mathcal{L}}_{\eta} f(x)\right| \leqslant c_{1}\left(\eta^{2-\alpha}+\eta^{\alpha}\right) .
$$


Hence $\overline{\mathcal{L}} f$ is the uniform limit of $\overline{\mathcal{L}}_{\eta}$ as $\eta \rightarrow 0$, and it follows that $\overline{\mathcal{L}} f \in C_{b}\left(\mathbb{R}^{d}\right)$ for any $f \in C_{c}^{2}\left(\mathbb{R}^{d}\right)$.

By the bounded convergence theorem,

$$
\lim _{n \rightarrow \infty} \hat{\mathbb{E}}\left[\left|\int_{s}^{t} \overline{\mathcal{L}} f\left(X_{r}^{n}\right) d r-\int_{s}^{t} \overline{\mathcal{L}} f\left(X_{r}\right) d r\right|\right]=0
$$

which together with (2.14) yields

$$
\lim _{n \rightarrow \infty} \hat{\mathbb{E}}\left[\left|\int_{s}^{t} \mathcal{L}_{n} f\left(X_{r}^{n}\right) d r-\int_{s}^{t} \overline{\mathcal{L}} f\left(X_{r}\right) d r\right|\right]=0 .
$$

Putting all the estimates above into (2.18), and letting $n \rightarrow \infty$, we obtain

$$
\hat{\mathbb{E}}\left[\left(f\left(X_{t}\right)-f\left(X_{s}\right)-\int_{s}^{t} \overline{\mathcal{L}} f\left(X_{r}\right) d r\right) G\left(X_{s_{1}}, \cdots X_{s_{k}}\right)\right]=0 .
$$

This shows that $\tilde{\mathbb{P}}_{\mathbf{0}}$ is a solution to the martingale problem $\left(\overline{\mathcal{L}}, C_{c}^{2}\left(\mathbb{R}^{d}\right)\right)$ and clearly $\tilde{\mathbb{P}}_{\mathbf{0}}\left(X_{0}=\mathbf{0}\right)=1$. This completes the proof.

Remark 2.5. As the above proofs show, we can replace the balanced condition (1.1) by the following slightly weaker condition: there is some large $r_{0} \geqslant 1$ so that for all $r \geqslant r_{0}$ and $x \in \mathbb{Z}^{d}$,

$$
\sum_{z \in \mathbb{Z}_{0}^{d}:|z| \leqslant r} z \kappa(x, z) /|z|^{d+\alpha}=0
$$

Note that under (2.19) the generator of the process $X$ can be written as

$$
\mathcal{L} f(x)=\sum_{z \in \mathbb{Z}_{0}^{d}:|z| \leqslant r}(f(x+z)-f(x)-\nabla f(x) \cdot z) \frac{\kappa(x, z)}{|z|^{d+\alpha}}+\sum_{z \in \mathbb{Z}_{0}^{d}:|z|>r}(f(x+z)-f(x)) \frac{\kappa(x, z)}{|z|^{d+\alpha}}
$$

for all $f \in B_{b}\left(\mathbb{Z}^{d}\right)$ and $r \geqslant r_{0}$. This indicates that the process $X$ is almost driftless in large scale, and so the drift term does not contribute to the scaling process. On the other hand, when $\alpha \in(0,1)$ the tail of long range jumps for the process $X$ is up to the order $r^{-\alpha}$, which would dominate the drift term when we do the scaling. Based on this observation, we can expect that, when $\alpha \in(0,1)$, the invariance principle still holds without the balanced condition (1.1) (or (2.19)). The details are given in the next subsection.

2.2. Non-balanced case for $\alpha \in(0,1)$. In this subsection, we restrict ourselves to the case $0<\alpha<1$, and obtain similar results as in the previous subsection but without the balanced condition (1.1).

Assumption (B1*). There exist constants $\theta \in(0,1), C_{1}>0$ and $R_{0} \geqslant 1$ such that for every $R>R_{0}$ and $r \in\left[R^{\theta}, R\right]$,

$$
\sup _{x \in B(\mathbf{0}, 2 R)} \sum_{z \in \mathbb{Z}_{0}^{d}:|z| \leqslant r} \frac{\kappa(x, z)}{|z|^{d+\alpha-1}} \leqslant C_{1} r^{1-\alpha}
$$

and

$$
\sup _{x \in B(\mathbf{0}, 2 R)} \sum_{z \in \mathbb{Z}^{d:|z|>r}} \frac{\kappa(x, z)}{|z|^{d+\alpha}} \leqslant C_{1} r^{-\alpha}
$$

Clearly, condition (2.20) implies condition (2.2), while (2.21) is the same as (2.3) in Assumption (B1).

The following result corresponds to Theorem 2.4 in the balanced conductance case for $\alpha \in(0,2)$.

Theorem 2.6. Let $0<\alpha<1$. Suppose that Assumptions (B1*) and (B2) hold, and the solution of the martingale problem for $\left(\overline{\mathcal{L}}, C_{c}^{2}\left(\mathbb{R}^{d}\right)\right)$ defined by (1.4) is unique. Then the conclusion of Theorem 2.4 holds. 
Proof. In the proof of Theorem 2.4 and related lemmas, there are three places where condition (1.1) is used. Below, we explain how to modify the corresponding parts of the proof. The rest part goes through exactly as that for Theorem 2.4.

The first part is the estimates of (2.5) in the proof of Lemma 2.1. In the current non-balanced case,

$$
\begin{aligned}
\mathcal{L} f_{x, r}(y) & =\sum_{z \in \mathbb{Z}_{0}^{d}:|z| \leqslant r}\left(f_{x, r}(y+z)-f_{x, r}(y)\right) \frac{\kappa(y, z)}{|z|^{d+\alpha}}+\sum_{z \in \mathbb{Z}^{d:|z| \geqslant r}}\left(f_{x, r}(y+z)-f_{x, r}(y)\right) \frac{\kappa(y, z)}{|z|^{d+\alpha}} \\
& =: I_{1, r}+I_{2, r} .
\end{aligned}
$$

By (2.20), for every for every $R>R_{0}, r \in\left[R^{\theta}, R\right], x \in B(\mathbf{0}, R)$ and $y \in B(x, r)$,

$$
\left|I_{1, r}\right| \leqslant\left\|\nabla f_{x, r}\right\|_{\infty} \sum_{z \in \mathbb{Z}_{0}^{d}:|z| \leqslant r} \frac{\kappa(y, z)}{|z|^{d+\alpha-1}} \leqslant c_{2} r^{-1} \sup _{y \in B(\mathbf{0}, 2 R)} \sum_{z \in \mathbb{Z}_{\mathbf{0}}^{d}:|z| \leqslant r} \frac{\kappa(y, z)}{|z|^{d+\alpha-1}} \leqslant c_{3} r^{-\alpha} .
$$

By the same way as that in the proof of Lemma 2.1, we have $\left|I_{2, r}\right| \leqslant c_{4} r^{-\alpha}$ for all $x \in B(\mathbf{0}, R)$ and $y \in B(x, r)$. Thus (2.6) holds and, consequently, (2.4) holds.

The second part is (2.11) and (2.12) in the proof of Lemma 2.2. In the non-balanced case, estimate (2.11) can be done in the following way. For $n$ large enough, in view of (2.1), (2.20) and (2.21),

$$
\begin{aligned}
\left|\mathcal{L}_{n} f(x)\right| & \leqslant n^{-d} \sum_{z \in n^{-1} \mathbb{Z}_{0}^{d}:|z| \leqslant 1}\|\nabla f\|_{\infty}|z| \frac{\kappa(n x, n z)}{|z|^{d+\alpha}}+n^{-d} \sum_{z \in n^{-1} \mathbb{Z}^{d}:|z| \geqslant 1} 2\|f\|_{\infty} \frac{\kappa(n x, n z)}{|z|^{d+\alpha}} \\
& \leqslant n^{\alpha-1}\|\nabla f\|_{\infty} \sum_{z \in \mathbb{Z}_{0}^{d}:|z| \leqslant n} \frac{\kappa(n x, z)}{|z|^{d+\alpha-1}}+2 n^{\alpha}\|f\|_{\infty} \sum_{z \in \mathbb{Z}^{d}:|z|>n} \frac{\kappa(n x, z)}{|z|^{d+\alpha}} \\
& \leqslant C_{1}\|\nabla f\|_{\infty}+2 C_{1}\|f\|_{\infty} .
\end{aligned}
$$

Hence (2.11) holds. On the other hand, the fourth inequality of (2.12) should be replaced by

$$
4\|f\|_{\infty} n^{\alpha}|n x|^{-1} \sum_{z \in \mathbb{Z}_{0}^{d}:|z| \leqslant 2 n|x|} \frac{\kappa(n x, z)}{|z|^{d+\alpha-1}} .
$$

Then, by (2.20), the end estimate in (2.12) holds.

The third place is the estimates for $I_{4}^{n, R, \eta}$ and $I_{6}^{n, R, \eta}$ in (2.16). Without the balanced condition, we can not insert the item $\nabla f(x) \cdot z$ into the definitions of $I_{4}^{n, R, \eta}$ and $I_{6}^{n, R, \eta}$. Since $0<\alpha<1$, we do it directly. That is,

$$
I_{4}^{n, R, \eta}=n^{-d} \sup _{x \in n^{-1} \mathbb{Z}^{d}:|x| \leqslant R}\left|\sum_{z \in n^{-1} \mathbb{Z}_{0}^{d}:|z| \leqslant \eta}(f(x+z)-f(x)) \frac{\kappa(n x, n z)}{|z|^{d+\alpha}}\right| .
$$

Then, by the same argument as $(2.22)$, we get

$$
\lim _{\eta \downarrow 0} \limsup _{n \rightarrow \infty} I_{4}^{n, R, \eta} \leqslant c_{5} \lim _{\eta \downarrow 0} \eta^{1-\alpha}=0 .
$$

Similarly, we have

$$
\lim _{\eta \downarrow 0} \limsup _{n \rightarrow \infty} I_{6}^{n, R, \eta} \leqslant c_{6} \lim _{\eta \downarrow 0}\left(\eta^{1-\alpha}+\eta^{\alpha}\right)=0 .
$$

With the above modifications, the proof of Lemma 2.3 goes through. Hence the conclusion of Theorem 2.4 holds under the condition of this theorem.

2.3. Balanced case for $\alpha=2$. In this subsection, we consider the case $\alpha=2$ under the balanced condition (1.1). We will make the following two assumptions instead of Assumptions (B1) and (B2), respectively. 
Assumption (C1). There exist constants $\theta \in(0,1), C_{1}>0$ and $R_{0} \geqslant 1$ such that for every $R>R_{0}$ and $r \in\left[R^{\theta}, R\right]$,

$$
\sup _{x \in B(\mathbf{0}, 2 R)} \sum_{z \in \mathbb{Z}^{d}: 1 \leqslant|z| \leqslant r} \frac{\kappa(x, z)}{|z|^{d}} \leqslant C_{1} \log (1+r),
$$

and

$$
\sup _{x \in B(\mathbf{0}, 2 R)} \sum_{z \in \mathbb{Z}^{d}:|z|>r} \frac{\kappa(x, z)}{|z|^{d+2}} \leqslant C_{1} r^{-2}
$$

Assumption (C2). For any $n \geqslant 1$, there exists a function $\Phi_{n}(x, z): n^{-1} \mathbb{Z}^{d} \times n^{-1} \mathbb{Z}_{\mathbf{0}}^{d} \rightarrow(0, \infty)$ with $\Phi_{n}(x, z)=\Phi_{n}(x,-z)$ for all $x \in n^{-1} \mathbb{Z}^{d}$ and $z \in n^{-1} \mathbb{Z}_{\mathbf{0}}^{d}$, so that $\sup _{n \geqslant 1}\left\|\Phi_{n}\right\|_{\infty}<\infty$, and for any integer $R \geqslant 1$ and any $f \in C_{c}^{2}\left(\mathbb{R}^{d}\right)$,

$$
\lim _{n \rightarrow \infty} \frac{n^{2}}{\log (1+n)} \sup _{\substack{x \in \mathbb{Z}^{d}: \\|x| \leqslant n R}}\left|\sum_{\substack{z \in \mathbb{Z}^{d}: \\ 1 \leqslant|z| \leqslant n}}\left(f\left(\frac{x+z}{n}\right)-f\left(\frac{x}{n}\right)\right)\left(\frac{\kappa(x, z)-\Phi_{n}(x / n, z / n)}{|z|^{d+2}}\right)\right|=0,
$$

and that for any integer $R \geqslant 1$ and $1 \leqslant i, j \leqslant d$,

$$
\lim _{n \rightarrow \infty} \sup _{x \in n^{-1} \mathbb{Z}^{d}:|x| \leqslant R}\left|\frac{1}{\log (1+n)} \sum_{z \in \mathbb{Z}_{0}^{d}:|z| \leqslant n} \frac{z_{i} z_{j} \Phi_{n}(x, z / n)}{|z|^{\mid+2}}-a_{i j}\right|=0
$$

for some constant matrix $A:=\left(a_{i j}\right)_{1 \leqslant i, j \leqslant d}$ on $\mathbb{R}^{d}$.

Corresponding to Theorem 2.4, we have the following result.

Theorem 2.7. Let $\alpha=2$. Assume that the balanced condition (1.1), and Assumptions (C1) and (C2) hold. Then the conclusion of Theorem 2.4 holds with

$$
\overline{\mathcal{L}} f(x):=\frac{1}{2} \sum_{1 \leqslant i, j \leqslant d} a_{i j} \frac{\partial^{2} f(x)}{\partial x_{i} \partial x_{j}}, \quad f \in C_{c}^{2}\left(\mathbb{R}^{d}\right) .
$$

Proof. The proof follows from that of Theorem 2.4 with some necessary modifications. For the convenience of the reader, we highlight all main differences here.

(1) First, under Assumption (C1), there is a constant $c>0$ that depends only on the constant $C_{1}$ in Assumption (C1) so that for every $R>R_{0}, r \in\left[R^{\theta}, R\right], x \in B(\mathbf{0}, R)$ and $t>0$,

$$
\mathrm{P}_{x}\left(\tau_{B(x, r)} \leqslant t\right) \leqslant c t r^{-2} \log (1+r) .
$$

Consequently, $\left\{\mathbb{P}_{\mathbf{0}}^{n}\right\}_{n=1}^{\infty}$ is tight in $\mathscr{D}\left([0, T] ; \mathbb{R}^{d}\right)$ for any $T>0$.

Under Assumption (C1), we have that for every $f \in C_{c}^{2}\left(\mathbb{R}^{d}\right)$,

$$
\sup _{n \geqslant 1} \sup _{x \in n^{-1} \mathbb{Z}^{d}}\left|\mathcal{L}_{n} f(x)\right|<\infty, \quad \lim _{R \rightarrow \infty} \limsup _{n \rightarrow \infty} \sup _{x \in n^{-1} \mathbb{Z}^{d}:|x| \geqslant R}\left|\mathcal{L}_{n} f(x)\right|=0 .
$$

The proofs of the above conclusions are similar to these of Lemmas 2.1 and 2.2, so they are omitted.

(2) Next, we claim that under Assumptions (C1) and (C2), for any $f \in C_{c}^{3}\left(\mathbb{R}^{d}\right)$,

$$
\lim _{n \rightarrow \infty} \sup _{x \in n^{-1} \mathbb{Z}^{d}}\left|\mathcal{L}_{n} f(x)-\overline{\mathcal{L}} f(x)\right|=0,
$$

where the operator $\overline{\mathcal{L}}$ is defined by (2.27). Indeed, for any $n \geqslant 1$ and $x \in n^{-1} \mathbb{Z}^{d}$, define

$$
\begin{aligned}
\mathcal{L}_{n, 1} f(x): & =\frac{1}{n^{d} \log (1+n)} \sum_{z \in n^{-1} \mathbb{Z}^{d: 0<|z| \leqslant 1}}(f(x+z)-f(x)) \frac{\kappa(n x, n z)}{|z|^{d+2}} \\
& =\frac{n^{2}}{\log (1+n)} \sum_{z \in \mathbb{Z}^{d}: 1 \leqslant|z| \leqslant n}\left(f\left(x+\frac{z}{n}\right)-f(x)\right) \frac{\kappa(n x, z)}{|z|^{d+2}},
\end{aligned}
$$




$$
\begin{aligned}
\overline{\mathcal{L}}_{n, 1} f(x): & =\frac{1}{n^{d} \log (1+n)} \sum_{z \in n^{-1} \mathbb{Z}^{d}: 0<|z| \leqslant 1}(f(x+z)-f(x)) \frac{\Phi_{n}(x, z)}{|z|^{d+2}} \\
& =\frac{n^{2}}{\log (1+n)} \sum_{z \in \mathbb{Z}^{d}: 1 \leqslant|z| \leqslant n}\left(f\left(x+\frac{z}{n}\right)-f(x)\right) \frac{\Phi_{n}(x, z / n)}{|z|^{d+2}} .
\end{aligned}
$$

For every $R>1$,

$$
\begin{aligned}
\sup _{x \in n^{-1} \mathbb{Z}^{d}}\left|\mathcal{L}_{n} f(x)-\overline{\mathcal{L}} f(x)\right| \leqslant & \sup _{x \in n^{-1} \mathbb{Z}^{d}:|x| \leqslant R}\left|\mathcal{L}_{n, 1} f(x)-\overline{\mathcal{L}} f(x)\right|+\sup _{x \in n^{-1} \mathbb{Z}^{d}:|x| \leqslant R}\left|\mathcal{L}_{n} f(x)-\mathcal{L}_{n, 1} f(x)\right| \\
& +\sup _{x \in n^{-1} \mathbb{Z}^{d}:|x|>R}\left|\mathcal{L}_{n} f(x)\right|+\sup _{x \in \mathbb{R}^{d}:|x|>R}|\overline{\mathcal{L}} f(x)| \\
= & : I_{1}^{n, R}+I_{2}^{n, R}+I_{3}^{n, R}+I_{4}^{R} .
\end{aligned}
$$

According to (2.25), for every $R>1$,

$$
\lim _{n \rightarrow \infty} \sup _{x \in n^{-1} \mathbb{Z}^{d}:|x| \leqslant R}\left|\mathcal{L}_{n, 1} f(x)-\overline{\mathcal{L}}_{n, 1} f(x)\right|=0 .
$$

On the other hand, for any $f \in C_{c}^{3}\left(\mathbb{R}^{d}\right)$ and $x \in n^{-1} \mathbb{Z}^{d}$ with $|x| \leqslant R$

$$
\begin{aligned}
\left|\overline{\mathcal{L}}_{n, 1} f(x)-\overline{\mathcal{L}} f(x)\right| & =\left|\frac{n^{2}}{\log (1+n)} \sum_{z \in \mathbb{Z}^{d: 1 \leqslant|z| \leqslant n}}\left(f\left(x+\frac{z}{n}\right)-f(x)-\nabla f(x) \cdot \frac{z}{n}\right) \frac{\Phi_{n}(x, z / n)}{|z|^{d+2}}-\overline{\mathcal{L}} f(x)\right| \\
\leqslant & \left|\frac{1}{2 \log (1+n)} \sum_{z \in \mathbb{Z}^{d}: 1 \leqslant|z| \leqslant n}\left\langle\nabla^{2} f(x), z \otimes z\right\rangle \frac{\Phi_{n}(x, z / n)}{|z|^{d+2}}-\overline{\mathcal{L}} f(x)\right| \\
& +\frac{c_{1}\left\|\nabla^{3} f\right\|_{\infty}}{n \log (1+n)} \sum_{z \in \mathbb{Z}^{d: 1 \leqslant|z| \leqslant n}} \frac{|z|^{3}}{|z|^{d+2}} .
\end{aligned}
$$

Here in the equality above we can add the gradient term $\nabla f(x) \cdot \frac{z}{n}$ in the summation, thanks to the property that $\Phi_{n}(x, z)=\Phi_{n}(x,-z)$ for all $x \in n^{-1} \mathbb{Z}^{d}, z \in n^{-1} \mathbb{Z}_{\mathbf{0}}^{d}$ and $n \geqslant 1$; and the inequality above follows from the Taylor formula and the fact that $\sup _{n \geqslant 1}\left\|\Phi_{n}\right\|_{\infty}<\infty$. Hence by (2.26) and the definition of the operator $\overline{\mathcal{L}}$ given by $(2.27)$, we can see that

$$
\lim _{n \rightarrow \infty} \sup _{x \in n^{-1} \mathbb{Z}^{d}:|x| \leqslant R}\left|\overline{\mathcal{L}}_{n, 1} f(x)-\overline{\mathcal{L}} f(x)\right|=0 .
$$

Therefore for any $R>1$,

$$
\lim _{n \rightarrow \infty} I_{1}^{n, R}=0
$$

By (2.24), for any $R>1$,

$$
\limsup _{n \rightarrow \infty} I_{2}^{n, R} \leqslant 2\|f\|_{\infty} \limsup _{n \rightarrow \infty} \sup _{x \in \mathbb{Z}^{d}:|x| \leqslant n R} \frac{n^{2}}{\log (1+n)} \sum_{|z| \geqslant n} \frac{\kappa(x, z)}{|z|^{d+2}}=0 .
$$

According to (2.28),

$$
\lim _{R \rightarrow \infty} \limsup _{n \rightarrow \infty} I_{3}^{n, R}=0 .
$$

By the definition of the operator $\overline{\mathcal{L}}$ given by $(2.27)$ again, it is obvious that

$$
\lim _{R \rightarrow \infty} I_{4}^{R}=0 .
$$

Therefore, (2.29) is a consequence of all estimates above, by first letting $n \rightarrow \infty$ and then taking $R \rightarrow \infty$.

(3) According to (2.26), $A:=\left(a_{i j}\right)_{1 \leqslant i, j \leqslant d}$ given in Assumption (C2) is non-negative definite. Note that the solution of the martingale problem for $\left(\overline{\mathcal{L}}, C_{c}^{2}\left(\mathbb{R}^{d}\right)\right)$ defined by $(2.27)$ is always unique, since it corresponds to Brownian motion with a deterministic covariance matrix $A$. With (1) and (2) at hand, we can follow the argument of the proof of Theorem 2.4 to obtain the desired assertion. 


\section{RANDOM WALKS IN BALANCED RANDOM ENVIRONMENTS}

Proof of Theorem 1.2(i). First, we claim that Assumptions (A0) and (A1) imply that for a.s. $\omega \in \Omega$, Assumption (B1) holds, and that for every integer $R \geqslant 1$, rational constant $\varepsilon>0$ and $f \in C_{c}^{2}\left(\mathbb{R}^{d}\right)$,

$$
\lim _{n \rightarrow \infty} n^{\alpha} \sup _{\substack{x \in \mathbb{Z}^{d}: \\|x| \leqslant n R}}\left|\sum_{\substack{z \in \mathbb{Z}^{d} \\ n \varepsilon<|z|<n / \varepsilon}}\left(f\left(\frac{x+z}{n}\right)-f\left(\frac{x}{n}\right)\right)\left(\frac{\kappa(x, z)-\mathbb{E}[\kappa(x, z)]}{|z|^{d+\alpha}}\right)\right|=0 .
$$

The proof is mainly based on that of [6, Proposition 5.6]. For the convenience of the reader, we give the details here. Set $J(x, z):=\mathbb{E}[\kappa(x, z)]$. For $x \in \mathbb{Z}^{d}, R, \delta, \varepsilon>0$ and $h: \mathbb{Z}^{d} \times \mathbb{Z}^{d} \rightarrow \mathbb{R}$, define

$$
\begin{aligned}
q_{1}(x, \delta, h, \varepsilon) & :=\mathbb{P}\left(\left|\sum_{\substack{z \in \mathbb{Z}^{d}: \\
n \varepsilon \leqslant|z| \leqslant n / \varepsilon}} h(x, z) \frac{(\kappa(x, z)-J(x, z))}{|z|^{d+\alpha}}\right|>\delta(n \varepsilon)^{-\alpha}\right), \\
q_{2}(x, R, \delta) & :=\mathbb{P}\left(\left|\sum_{z \in \mathbb{Z}_{0}^{d}:|z| \leqslant R}(\kappa(x, z)-J(x, z))\right|>\delta R^{d}\right), \\
q_{3}(x, R, \delta) & :=\mathbb{P}\left(\left|\sum_{z \in \mathbb{Z}_{0}^{d}:|z| \leqslant R} \frac{(\kappa(x, z)-J(x, z))}{|z|^{d+\alpha-2}}\right|>\delta R^{2-\alpha}\right) .
\end{aligned}
$$

Note that for a series of independent random variables $\left\{\eta_{i}\right\}_{1 \leqslant i \leqslant n}$ with $\mathbb{E}\left[\eta_{i}\right]=0$ for all $1 \leqslant i \leqslant n$ and $M:=\sup _{1 \leqslant i \leqslant n} \mathbb{E}\left[\left|\eta_{i}\right|^{q}\right]<\infty$ for some $q \geqslant 1$, by the Burkholder-Davis-Gundy inequality,

$$
\mathbb{E}\left[\left|\sum_{i=1}^{n} \eta_{i}\right|^{q}\right] \leqslant c_{0} \mathbb{E}\left[\left(\sum_{i=1}^{n} \eta_{i}^{2}\right)^{q / 2}\right] \leqslant c_{1} \max \left\{n^{q / 2-1}, 1\right\} \sum_{i=1}^{n} \mathbb{E}\left[\left|\eta_{i}\right|^{q}\right] \leqslant c_{2} n^{\max \{q / 2,1\}} M,
$$

where $c_{0}, c_{1}, c_{2}$ are positive constants that depend only on $q$.

For every $m \in \mathbb{R}_{+}$, let

$$
S_{m}(i):=\mathbb{E}\left[\left(\sum_{z \in \mathbb{Z}_{0}^{d}:|z| \leqslant 2^{i}} \frac{(\kappa(x, z)-J(x, z))}{|z|^{d+\alpha-2}}\right)^{m}\right]=2^{m} \mathbb{E}\left[\left(\sum_{j=1}^{i} \xi(j)\right)^{m}\right],
$$

where

$$
\xi(j)=\sum_{z \in \mathbb{Z}_{+, *}^{d}: 2^{j-1}<|z| \leqslant 2^{j}} \frac{(\kappa(x, z)-J(x, z))}{|z|^{d+\alpha-2}} .
$$

Recall that $J(x, z)=\mathbb{E}[\kappa(x, z)]$ and $\left\{\kappa(x, z): x \in \mathbb{Z}^{d}, z \in \mathbb{Z}_{+, *}^{d}\right\}$ are independent. For every $m \in[2, p]$, by (3.2), there is a constant $c_{3}>0$ depending only on $m$ and $d$ so that

$$
\begin{aligned}
\mathbb{E}\left[\mid \sum_{z \in \mathbb{Z}_{+, *}^{d}:|z|=k} \frac{\kappa(x, z)-J(x, z)}{\left.\left.|z|^{d+\alpha-2}\right|^{m}\right]} \leqslant c_{3} k^{-m(d+\alpha-2)} k^{\frac{(d-1) m}{2}} \sup _{x \in \mathbb{Z}^{d}, z \in \mathbb{Z}_{\mathbf{0}}^{d}} \mathbb{E}\left[\kappa(x, z)^{m}\right]\right. \\
=c_{3} k^{\frac{m(3-2 \alpha-d)}{2}} \sup _{x \in \mathbb{Z}^{d}, z \in \mathbb{Z}_{\mathbf{0}}^{d}} \mathbb{E}\left[\kappa(x, z)^{m}\right] .
\end{aligned}
$$

Hence by (3.2) again,

$$
\begin{aligned}
& \mathbb{E}\left[|\xi(j)|^{m}\right]=\mathbb{E}\left[\left|\sum_{k=2^{j-1}+1}^{2^{j}} \sum_{z \in \mathbb{Z}_{+, *}^{d}:|z|=k} \frac{\kappa(x, z)-J(x, z)}{|z|^{d+\alpha-2}}\right|^{m}\right] \\
& \leqslant c_{4} 2^{\frac{j m}{2}} 2^{\frac{j m(3-2 \alpha-d)}{2}} \sup _{x \in \mathbb{Z}^{d}, z \in \mathbb{Z}_{\mathbf{0}}^{d}} \mathbb{E}\left[\kappa(x, z)^{m}\right]=c_{4} 2^{\frac{j m(4-d-2 \alpha)}{2}} \sup _{x \in \mathbb{Z}^{d}, z \in \mathbb{Z}_{\mathbf{0}}^{d}} \mathbb{E}\left[\kappa(x, z)^{m}\right],
\end{aligned}
$$


where $c_{4}>0$ is a constant that depends only on $m$ and $d$. Consequently, according to the first inequality in (3.2) and the Hölder inequality as well as Assumption (A1)(i), we know that for each $\varepsilon \in(0, d-4+2 \alpha)$ (thanks to the assumption that $d>4-2 \alpha$ )

$$
\begin{aligned}
\sup _{i \geqslant 0}\left|S_{p}(i)\right| & \leqslant c_{5} \sup _{i \geqslant 0} \mathbb{E}\left[\left|\sum_{j=1}^{i} \xi(j)^{2}\right|^{p / 2}\right] \leqslant c_{5} \sup _{i \geqslant 0} \mathbb{E}\left[\left(\sum_{j=1}^{i} 2^{\frac{\varepsilon j p}{2}}|\xi(j)|^{p}\right)\left(\sum_{j=1}^{i} 2^{-\frac{\varepsilon j p}{p-2}}\right)^{p / 2-1}\right] \\
& \leqslant c_{6} \sup _{i \geqslant 0} \sum_{j=1}^{i} 2^{\frac{\varepsilon j p}{2}} \mathbb{E}\left[|\xi(j)|^{p}\right] \leqslant c_{7} \sum_{j=1}^{\infty} 2^{\frac{j p(4+\varepsilon-d-2 \alpha)}{2}}<\infty .
\end{aligned}
$$

Then, using the Markov inequality and the fact that $p>\frac{d+1}{2-\alpha}$, we can find a constant $\theta \in(0,1)$ such that

$$
\sum_{R=1}^{\infty} \sum_{x \in B(\mathbf{0}, 2 R) \cap \mathbb{Z}^{d}} q_{3}\left(x, R^{\theta}, \delta\right) \leqslant c_{8}(\delta) \sum_{R=1}^{\infty} R^{d-(2-\alpha) \theta p}<\infty .
$$

Similarly, since $p>\frac{2(d+1)}{d}$, we can also show that

$$
\sum_{R=1}^{\infty} \sum_{x \in B(\mathbf{0}, 2 R) \cap \mathbb{Z}^{d}} \sum_{r=R^{\theta} / 2}^{\infty} q_{2}(x, r, \delta) \leqslant c_{9}(\delta) \sum_{R=1}^{\infty} R^{d} \sum_{r=R^{\theta}}^{\infty} r^{-d p / 2}<\infty .
$$

Thus, according to (3.4), (3.5) and the Borel-Cantelli lemma, for a.s. $\omega \in \Omega$ there exists $R_{0}(\omega) \geqslant 2$ such that for all $R>R_{0}(\omega), x \in B(\mathbf{0}, 2 R) \cap \mathbb{Z}^{d}$ and $r \geqslant R^{\theta} / 2$,

$$
\sum_{z \in \mathbb{Z}_{0}^{d}:|z| \leqslant R^{\theta}} \frac{\kappa(x, z)}{|z|^{d+\alpha-2}} \leqslant c_{10} R^{\theta(2-\alpha)} \text { and } \sum_{z \in \mathbb{Z}_{0}^{d}:|z| \leqslant r} \kappa(x, z) \leqslant c_{11} r^{d} .
$$

Therefore, for $R \geqslant R_{0}(\omega)$, every $r \in\left[R^{\theta}, R\right]$ and $x \in B(0,2 R)$,

$$
\begin{aligned}
\sum_{1 \leqslant|z| \leqslant r} \frac{\kappa(x, z)}{|z|^{d+\alpha-2}} & \leqslant \sum_{1 \leqslant|z| \leqslant R^{\theta}} \frac{\kappa(x, z)}{|z|^{d+\alpha-2}}+\sum_{R^{\theta} \leqslant|z| \leqslant r} \frac{\kappa(x, z)}{|z|^{d+\alpha-2}} \leqslant c_{10} R^{\theta(2-\alpha)}+\sum_{i=\left[\frac{\theta \log R}{\log 2}\right] 2^{i} \leqslant|z|<2^{i+1}} \frac{\kappa(x, z)}{|z|^{d+\alpha-2}} \\
& \leqslant c_{10} R^{\theta(2-\alpha)}+c_{11} \sum_{i=\left[\frac{\log r}{\log 2}\right]}^{\left[\frac{\log r}{\log 2}\right]} 2^{-i(d+\alpha-2)} 2^{(i+1) d} \leqslant c_{12} r^{2-\alpha},
\end{aligned}
$$

and

$$
\begin{aligned}
\sum_{|z|>r} \frac{\kappa(x, z)}{|z|^{d+\alpha}} & \leqslant \sum_{i=\left[\frac{\log r}{\log 2}\right]}^{\infty} \sum_{2^{i} \leqslant|z|<2^{i+1}} \frac{\kappa(x, z)}{|z|^{d+\alpha}} \leqslant \sum_{i=\left[\frac{\log r}{\log 2}\right]}^{\infty} 2^{-i(d+\alpha)} \sum_{z \in \mathbb{Z}_{\mathbf{0}}^{d}:|z|<2^{i+1}} \kappa(x, z) \\
& \leqslant c_{11} \sum_{i=\left[\frac{\log r}{\log 2}\right]}^{\infty} 2^{-i(d+\alpha)} 2^{(i+1) d} \leqslant c_{12} r^{-\alpha} .
\end{aligned}
$$

This shows that Assumption (B1) holds for a.s. $\omega \in \Omega$.

For any fixed $f \in C_{c}^{2}\left(\mathbb{R}^{d}\right)$ and $n \geqslant 1$, let

$$
f_{n}(x, z):=f\left(\frac{x+z}{n}\right)-f\left(\frac{x}{n}\right) .
$$

Then, for any $R \geqslant 1$ and $\varepsilon, \delta>0$,

$$
\sum_{n=1}^{\infty} \sum_{x \in B(\mathbf{0}, n R) \cap \mathbb{Z}^{d}} q_{1}\left(x, \delta, f_{n}, \varepsilon\right) \leqslant \sum_{n=1}^{\infty} \sum_{x \in B(\mathbf{0}, n R) \cap \mathbb{Z}^{d}} \delta^{-p}(\varepsilon n)^{\alpha p}(n \varepsilon)^{-p(d+\alpha)}
$$




$$
\begin{aligned}
& \times \mathbb{E}\left[\left|\sum_{z \in \mathbb{Z}^{d}: n \varepsilon \leqslant|z| \leqslant n / \varepsilon} f_{n}(x, z)(n \varepsilon)^{d+\alpha} \frac{(\kappa(x, z)-J(x, z))}{|z|^{d+\alpha}}\right|^{p}\right] \\
\leqslant & c_{13} \delta^{-p}(\varepsilon n)^{-p d}(n R)^{d}(n / \varepsilon)^{d p / 2}\left\|f_{n}\right\|_{\infty}^{p} \sup _{x \in \mathbb{Z}^{d}, z \in \mathbb{Z}_{0}^{d}} \mathbb{E}\left[\kappa(x, z)^{p}\right] \\
\leqslant & c_{14} \delta^{-p} \varepsilon^{-\frac{3 p d}{2}} R^{d}\|f\|_{\infty}^{p} \sum_{n=1}^{\infty} n^{-\left(\frac{p}{2}-1\right) d}<\infty .
\end{aligned}
$$

Here the first inequality is due to the Markov inequality, the second inequality follows from (1.1), (3.2) and Assumption (A1)(i), and in the last inequality we have used the fact that $p>\frac{2(d+1)}{d}$. By the Borel-Cantelli lemma, there is a subset $\Omega_{f}$ of $\Omega$ of full probability (which may depend on $f$ ) so that for every $\omega \in \Omega_{f}$ and every positive rational constants $\varepsilon, \delta$ and integer $R \geqslant 1$, there exists $N_{0}:=N_{0}\left(\omega, R, \delta, \varepsilon,\|f\|_{\infty}\right) \geqslant 1$ such that for every $n>N_{0}$ and $\omega \in \Omega_{f}$,

$$
n^{\alpha} \sup _{x \in B(\mathbf{0}, n R) \cap \mathbb{Z}^{d}}\left|\sum_{\substack{z \in \mathbb{Z}^{d}: \\ n \varepsilon \leqslant|z| \leqslant n / \varepsilon}} f_{n}(x, z) \frac{(\kappa(x, z)-J(x, z))}{|z|^{d+\alpha}}\right| \leqslant \delta \varepsilon^{-\alpha} .
$$

Taking $\delta \rightarrow 0$ in the inequality above, we can obtain that for every given $f \in C_{c}^{2}\left(\mathbb{R}^{d}\right)$, (3.1) holds for each $\omega \in \Omega_{f}$.

Now we are going to show that (3.1) holds on some subset $\Omega_{0}$ of $\Omega$ having full probability that is independent of $f$.

Let $\Upsilon \subset C_{c}^{2}\left(\mathbb{R}^{d}\right)$ be a countable dense subset in $\left(C_{c}\left(\mathbb{R}^{d}\right),\|\cdot\|_{\infty}\right)$, and set $\Omega_{1}:=\bigcap_{f \in \Upsilon} \Omega_{f}$. Then $\Omega_{1}$ is of full probability.

Given some $R>0$ and $\varepsilon>0$, define (for simplicity, we omit the parameter $\omega$ in $T_{n}(f)$ )

$$
T_{n}(f)=n^{\alpha} \sup _{x \in \mathbb{Z}^{d}:|x| \leqslant n R} Q_{n}(f, x), \quad f \in C_{c}^{2}\left(\mathbb{R}^{d}\right),
$$

where

$$
Q_{n}(f, x)=\left|\sum_{z \in \mathbb{Z}^{d}: n \varepsilon \leqslant|z| \leqslant n \varepsilon^{-1}}\left(f\left(\frac{x+z}{n}\right)-f\left(\frac{x}{n}\right)\right) \frac{\kappa(x, z)-J(x, z)}{|z|^{d+\alpha}}\right| .
$$

Therefore, for every $f, g \in C_{c}^{2}\left(\mathbb{R}^{d}\right)$,

$$
\begin{aligned}
\left|T_{n}(f)-T_{n}(g)\right| & \leqslant n^{\alpha} \sup _{x \in \mathbb{Z}^{d}:|x| \leqslant n R}\left|Q_{n}(f, x)-Q_{n}(g, x)\right| \\
& \leqslant n^{\alpha} \sup _{x \in \mathbb{Z}^{d}:|x| \leqslant n R}\left|\sum_{z \in \mathbb{Z}^{d}: n \varepsilon \leqslant|z| \leqslant n \varepsilon^{-1}}\left[(f-g)\left(\frac{x+z}{n}\right)-(f-g)\left(\frac{x}{n}\right)\right] \frac{\kappa(x, z)-J(x, z)}{|z|^{d+\alpha}}\right| \\
& \leqslant 2 n^{\alpha}\|f-g\|_{\infty}\left(\sup _{x \in \mathbb{Z}^{d}:|x| \leqslant n R} \sum_{|z| \geqslant n \varepsilon} \frac{\kappa(x, z)}{|z|^{d+\alpha}}+\sup _{x \in \mathbb{Z}^{d}:|x| \leqslant n R} \sum_{|z| \geqslant n \varepsilon} \frac{J(x, z)}{|z|^{d+\alpha}}\right) .
\end{aligned}
$$

By (3.6), there exists a subset $\Omega_{2} \subset \Omega$ having full probability such that for all $\omega \in \Omega_{1}$, integer $R \geqslant 1$, rational constant $\varepsilon>0$ and $n>N_{0}(\omega)$ large enough,

$$
\sup _{x \in \mathbb{Z}^{d}:|x| \leqslant n R} \sum_{|z|>n \varepsilon} \frac{\kappa(x, z)}{|z|^{d+\alpha}} \leqslant c_{15}(n \varepsilon)^{-\alpha} .
$$

Meanwhile, since $J(x, z)$ is uniformly bounded,

$$
\sup _{x \in \mathbb{Z}^{d}:|x| \leqslant n R} \sum_{|z|>n \varepsilon} \frac{J(x, z)}{|z|^{d+\alpha}} \leqslant c_{16}(n \varepsilon)^{-\alpha} .
$$


Combining all the estimates above together yields that for all $f, g \in C_{c}^{\infty}\left(\mathbb{R}^{d}\right)$ and $\omega \in \Omega_{1}$,

$$
\limsup _{n \rightarrow \infty}\left|T_{n}(f)-T_{n}(g)\right| \leqslant c_{17}(\varepsilon)\|f-g\|_{\infty},
$$

where $c_{17}$ is independent of $f, g$ and $n$.

For any $f \in C_{c}^{\infty}\left(\mathbb{R}^{d}\right)$, we can find a sequence $\left\{f_{k}\right\}_{k=1}^{\infty} \subset \Upsilon$ such that $\lim _{k \rightarrow \infty}\left\|f_{k}-f\right\|_{\infty}=0$. Let $\Omega_{0}:=\Omega_{1} \cap \Omega_{2}$. Obviously $\Omega_{0}$ has full probability. For every $\omega \in \Omega_{0}$,

$$
\lim _{n \rightarrow \infty} T_{n}(f) \leqslant \lim _{n \rightarrow \infty} T_{n}\left(f_{k}\right)+\limsup _{n \rightarrow \infty}\left|T_{n}(f)-T_{n}\left(f_{k}\right)\right| \leqslant c_{17}(\omega)\left\|f-f_{k}\right\|_{\infty},
$$

where in the second inequality we used the fact that (3.1) holds for every $\omega \in \Omega_{0}$ and every $g \in \Upsilon$. Then, letting $k \rightarrow \infty$ in the inequality above, we know that (3.1) is true for every $\omega \in \Omega$ and $f \in C_{c}^{2}\left(\mathbb{R}^{d}\right)$. Therefore, by (3.1) and Assumption (A1)(ii) (in particular, (1.3)), we conclude that

$$
\lim _{n \rightarrow \infty} n^{\alpha} \sup _{\substack{x \in \mathbb{Z}^{d} \\|x| \leqslant n R}}\left|\sum_{\substack{z \in \mathbb{Z}^{d}: \\ n \varepsilon<|z|<n / \varepsilon}}\left(f\left(\frac{x+z}{n}\right)-f\left(\frac{x}{n}\right)\right)\left(\frac{\kappa(x, z)-K\left(\frac{x}{n}, \frac{z}{n}\right)}{|z|^{d+\alpha}}\right)\right|=0
$$

holds for every $\omega \in \Omega_{0}$, rational constant $\varepsilon>0$ small enough, integer $R \geqslant 1$ large enough and any $f \in C_{c}^{2}\left(\mathbb{R}^{d}\right)$. In particular, this implies that (2.13) holds for every $\omega \in \Omega_{0}$, integer $R \geqslant 1$ large enough and any $f \in C_{c}^{2}\left(\mathbb{R}^{d}\right)$. Hence, Assumption (B2) holds for every $\omega \in \Omega_{0}$. The conclusion of Theorem 1.2(i) now follows from Theorem 2.4.

Proof of Theorem 1.2(ii). Suppose that Assumptions (A0) and (A2) hold. We will show that Assumptions $(\mathbf{C 1})$ and (C2) are satisfied for a.s. $\omega \in \Omega$. For any $x \in \mathbb{Z}^{d}$ and $z \in \mathbb{Z}_{\mathbf{0}}^{d}$, set $J(x, z):=\mathbb{E}[\kappa(x, z)]$. We set for $x \in \mathbb{Z}^{d}, R, \delta>0$ and $h: \mathbb{Z}^{d} \times \mathbb{Z}^{d} \rightarrow \mathbb{R}$,

$$
\begin{aligned}
& q_{4}(x, \delta, h):=\mathbb{P}\left(\left|\sum_{z \in \mathbb{Z}^{d}: 1 \leqslant|z| \leqslant n} h(x, z) \frac{(\kappa(x, z)-J(x, z))}{|z|^{d+2}}\right|>\delta \frac{\log (n+1)}{n^{2}}\right), \\
& q_{5}(x, R, \delta):=\mathbb{P}\left(\left|\sum_{z \in \mathbb{Z}_{0}^{d}:|z| \leqslant R} \frac{(\kappa(x, z)-J(x, z))}{|z|^{d}}\right|>\delta \log (R+1)\right) .
\end{aligned}
$$

For any fixed $f \in C_{c}^{2}\left(\mathbb{R}^{d}\right)$ and $n \geqslant 1$, let $f_{n}(x, z):=f\left(\frac{x+z}{n}\right)-f\left(\frac{x}{n}\right)$. Then for any $R, n \geqslant 1$, $x \in B(\mathbf{0}, n R) \cap \mathbb{Z}^{d}$ and $\delta>0$,

$$
\begin{aligned}
& q_{4}\left(x, \delta, f_{n}\right) \\
& \leqslant \mathbb{P}\left(\left|\sum_{z \in \mathbb{Z}_{+, *}^{d}: 1 \leqslant|z| \leqslant n} f_{n}(x, z) \frac{(\kappa(x, z)-J(x, z))}{|z|^{d+2}}\right|>\frac{\delta}{2} \frac{\log (1+n)}{n^{2}}\right) \\
& \quad+\mathbb{P}\left(\left|\sum_{z \in \mathbb{Z}_{+, *}^{d}: 1 \leqslant|z| \leqslant n} f_{n}(x,-z) \frac{(\kappa(x, z)-J(x, z))}{|z|^{d+2}}\right|>\frac{\delta}{2} \frac{\log (1+n)}{n^{2}}\right) \\
& \leqslant \mathbb{P}\left(\left|\sum_{z \in \mathbb{Z}_{+, *}^{d}: 1 \leqslant|z| \leqslant n}\left\langle\nabla^{2} f\left(x_{*}(z, n)\right), \frac{z}{|z|} \otimes \frac{z}{|z|}\right\rangle \frac{(\kappa(x, z)-J(x, z))}{|z|^{d}}\right|>\delta \log (n+1)\right) \\
& \quad+\mathbb{P}\left(\left|\sum_{z \in \mathbb{Z}_{+, *}^{d}: 1 \leqslant|z| \leqslant n}\left\langle\nabla^{2} f\left(x_{*}(-z, n)\right), \frac{z}{|z|} \otimes \frac{z}{|z|}\right\rangle \frac{(\kappa(x, z)-J(x, z))}{|z|^{d}}\right|>\delta \log (n+1)\right) .
\end{aligned}
$$

Here in the first inequality we used the balanced condition (1.1), in the second inequality we applied the Taylor formula and $x_{*}(z, n)=x / n+\theta_{0}(x, z, n) z / n$ with $\theta_{0}(x, z, n) \in[0,1]$ being a constant depending only on $x, z$ and $n$. By (1.7) in Assumption (A2)(i), for $c_{0}:=c_{*} /\left(2\left\|\nabla^{2} f\right\|_{\infty}\right)^{\eta}$,

$$
C_{*}:=\sup _{x \in \mathbb{Z}^{d}, z \in \mathbb{Z}_{\mathbf{0}}^{d}, n \geqslant 1} \mathbb{E} e^{c_{0}\left|\left\langle\nabla^{2} f\left(x_{*}(z, n)\right), \frac{z}{|z|} \otimes \frac{z}{|z|}\right\rangle(\kappa(x, z)-J(x, z))\right|^{\eta}}<\infty .
$$


This along with the Markov inequality implies that there exist constants $c_{1}, c_{2}>0$ such that for all $x \in \mathbb{Z}^{d}, z \in \mathbb{Z}_{\mathbf{0}}^{d}, n \geqslant 1$ and $t>0$,

$$
\begin{aligned}
& \mathbb{E}\left[\exp \left(t\left|\left\langle\nabla^{2} f\left(x_{*}(z, n)\right), \frac{z}{|z|} \otimes \frac{z}{|z|}\right\rangle(\kappa(x, z)-J(x, z))\right|\right)\right] \\
& =1+t \int_{0}^{\infty} e^{t r} \mathbb{P}\left(\left|\left\langle\nabla^{2} f\left(x_{*}(z, n)\right), \frac{z}{|z|} \otimes \frac{z}{|z|}\right\rangle(\kappa(x, z)-J(x, z))\right| \geqslant r\right) d r \\
& \leqslant 1+C_{*} t \int_{0}^{\infty} e^{t r-c_{0} r^{\eta}} d r \leqslant c_{1} \exp \left(c_{2} t^{\eta /(\eta-1)}\right) .
\end{aligned}
$$

Hence by (3.7), (3.8), $\eta \in(1,2)$, the independence of $\left\{\kappa(x, z): x \in \mathbb{Z}^{d}, z \in \mathbb{Z}_{+, *}^{d}\right\}$ (due to Assumption (A0)) and [3, (2.95) in Theorem 2.51, p. 45], we get

$$
q_{4}\left(x, \delta, f_{n}\right) \leqslant c_{3} \exp \left(-c_{4} \log ^{\eta}(1+n)\right) .
$$

We emphasize that the constants $c_{i}(i=1, \cdots, 4)$ above are independent of $R$ and $n$ (but may depend on $\|f\|_{\infty}$ and $\delta$ ). Hence, for any $R \geqslant 1$ and $\delta>0$,

$$
\sum_{n=1}^{\infty} \sum_{x \in B(\mathbf{0}, n R) \cap \mathbb{Z}^{d}} q_{4}\left(x, \delta, f_{n}\right) \leqslant c_{5} \sum_{n=1}^{\infty} \exp \left(-c_{6} \log ^{\eta}(1+n)\right)<\infty .
$$

Similarly, we can prove that for any $\theta \in(0,1)$,

$$
\sum_{R=1}^{\infty} \sum_{x \in B(\mathbf{0}, 2 R) \cap \mathbb{Z}^{d}} \sum_{r=R^{\theta}}^{R} q_{5}(x, r, \delta) \leqslant c_{7} \sum_{R=1}^{\infty} \exp \left(-c_{8} \log ^{\eta}(1+R)\right)<\infty .
$$

By the Borel-Cantelli lemma, for a.s $\omega \in \Omega$, there exists $R_{0}(\omega) \geqslant 1$ such that for all $R>R_{0}(\omega)$, $x \in B(\mathbf{0}, 2 R) \cap \mathbb{Z}^{d}$ and $r \in\left[R^{\theta}, R\right]$,

$$
\sum_{z \in \mathbb{Z}_{0}^{d}:|z| \leqslant r} \frac{\kappa(x, z)}{|z|^{d}} \leqslant c_{9} \log (1+r)
$$

By the proof of (3.5), we can show that for a.s. $\omega \in \Omega$ there exists $R_{0}(\omega) \geqslant 1$ such that for all $R>R_{0}(\omega), x \in B(\mathbf{0}, 2 R) \cap \mathbb{Z}^{d}$ and $r \geqslant R^{\theta} / 2$,

$$
\sum_{z \in \mathbb{Z}_{0}^{d}:|z| \leqslant r} \kappa(x, z) \leqslant c_{10} r^{d}
$$

which along with the same argument for (3.6) gives us that (2.24) holds for a.s. $\omega \in \Omega$. Thus, we know that Assumption (C1) holds for a.s. $\omega \in \Omega$.

To show that Assumption (C2) holds for a.s. $\omega \in \Omega$ as well, we return to (3.9). By the BorelCantelli lemma, there is a set $\Omega_{f} \subset \Omega$ of full probability such that for every $\omega \in \Omega_{f}$ and for every rational constant $\delta$ and integer $R \geqslant 1$, there exists an integer $N_{0}:=N_{0}\left(\omega, R, \delta,\|f\|_{\infty}\right) \geqslant 2$ such that for all $n>N_{0}$ and $x \in B(\mathbf{0}, n R) \cap \mathbb{Z}^{d}$,

$$
\frac{n^{2}}{\log (n+1)}\left|\sum_{z \in \mathbb{Z}^{d: 1 \leqslant|z| \leqslant n}} f_{n}(x, z) \frac{\kappa(x, z)-J(x, z)}{|z|^{d+2}}\right| \leqslant \delta .
$$

Taking $\delta \rightarrow 0$ in the inequality above, we can obtain that for every $\omega \in \Omega_{f}$ and every integer $R \geqslant 1$,

$$
\lim _{n \rightarrow \infty} \frac{n^{2}}{\log (1+n)} \sup _{\substack{x \in \mathbb{Z}^{d}: \\|x| \leqslant n R}}\left|\sum_{z \in \mathbb{Z}^{d}: 1 \leqslant|z| \leqslant n} f_{n}(x, z) \frac{\kappa(x, z)-\mathbb{E}[\kappa(x, z)]}{|z|^{d+2}}\right|=0 .
$$


It is known that there is a countable subset $\Upsilon$ of $C_{c}^{2}\left(\mathbb{R}^{d}\right)$ that is dense in $\left(C_{c}^{2}\left(\mathbb{R}^{d}\right),\|\cdot\|_{2, \infty}\right)$ (with $\left.\|f\|_{2, \infty}:=\|f\|_{\infty}+\|\nabla f\|_{\infty}+\left\|\nabla^{2} f\right\|_{\infty}\right)^{1}$. Set $\Omega_{1}:=\bigcap_{f \in \Upsilon} \Omega_{f}$. Clearly, $\Omega_{1}$ has full probability.

Given some $R>0$, define (for simplicity, we omit the parameter $\omega$ in $T_{n}(f)$ )

$$
T_{n}(f)=\frac{n^{2}}{\log (1+n)} \sup _{x \in \mathbb{Z}^{d}:|x| \leqslant n R} Q_{n}(f, x), \quad f \in C_{c}^{2}\left(\mathbb{R}^{d}\right),
$$

where

$$
Q_{n}(f, x)=\left|\sum_{z \in \mathbb{Z}^{d}: 1 \leqslant|z| \leqslant n}\left(f\left(\frac{x+z}{n}\right)-f\left(\frac{x}{n}\right)\right) \frac{\kappa(x, z)-J(x, z)}{|z|^{d+2}}\right| .
$$

Therefore, for every $f, g \in C_{c}^{2}\left(\mathbb{R}^{d}\right)$,

$$
\begin{aligned}
& \left|T_{n}(f)-T_{n}(g)\right| \\
& \leqslant \frac{n^{2}}{\log (1+n)} \sup _{x \in \mathbb{Z}^{d}:|x| \leqslant n R}\left|Q_{n}(f, x)-Q_{n}(g, x)\right| \\
& \leqslant \frac{n^{2}}{\log (1+n)} \sup _{x \in \mathbb{Z}^{d}:|x| \leqslant n R}\left|\sum_{z \in \mathbb{Z}^{d}: 1 \leqslant|z| \leqslant n}\left((f-g)\left(\frac{x+z}{n}\right)-(f-g)\left(\frac{x}{n}\right)\right) \frac{\kappa(x, z)-J(x, z)}{|z|^{d+2}}\right| \\
& \leqslant \frac{1}{2 \log (1+n)}\left\|\nabla^{2}(f-g)\right\|_{\infty}\left(\sup _{x \in \mathbb{Z}^{d}:|x| \leqslant n R} \sum_{1 \leqslant|z| \leqslant n} \frac{\kappa(x, z)}{|z|^{d+2}}+\sup _{x \in \mathbb{Z}^{d}:|x| \leqslant n R} \sum_{1 \leqslant|z| \leqslant n} \frac{J(x, z)}{|z|^{d+2}}\right),
\end{aligned}
$$

where in the last inequality we used (1.1) and the mean value theorem. By (3.10) we know there exists a set $\Omega_{2}$ of full probability such that for all $\omega \in \Omega_{2}$, any integer $R \geqslant 1$ and $n>N_{0}(\omega)$ large enough,

$$
\sup _{x \in \mathbb{Z}^{d:|x| \leqslant n R}} \sum_{|z| \leqslant n} \frac{\kappa(x, z)}{|z|^{d+2}} \leqslant c_{11} \log (1+n) .
$$

Meanwhile, since $J(x, z)$ is uniformly bounded,

$$
\sup _{x \in \mathbb{Z}^{d}:|x| \leqslant n R} \sum_{1 \leqslant|z| \leqslant n} \frac{J(x, z)}{|z|^{\mid+2}} \leqslant c_{12} \log (1+n) .
$$

Combining all the estimates above together yields that for all $f, g \in C_{c}^{2}\left(\mathbb{R}^{d}\right)$ and $\omega \in \Omega_{2}$,

$$
\limsup _{n \rightarrow \infty}\left|T_{n}(f)-T_{n}(g)\right| \leqslant c_{13}(\varepsilon)\left\|\nabla^{2}(f-g)\right\|_{\infty},
$$

where $c_{13}$ is independent of $f, g$ and $n$. With this assertion at hand, we can then follow the proof of Theorem 1.2(i) to deduce that (3.11) holds for all $f \in C_{c}^{2}\left(\mathbb{R}^{d}\right)$ and every $\omega \in \Omega_{0}:=\Omega_{1} \cap \Omega_{2}$. This implies that (2.25) holds for every $\omega \in \Omega_{0}$ with $\Phi_{n}(x, y):=J(n x, n z)$ for each $x \in n^{-1} \mathbb{Z}^{d}, z \in n^{-1} \mathbb{Z}_{\mathbf{0}}^{d}$. Clearly (1.8) implies that (2.26) holds for the function $\Phi_{n}(x, z)$ defined above. It is also obvious that $\Phi_{n}(x, z)=\Phi_{n}(x,-z)$ for $x \in n^{-1} \mathbb{Z}^{d}, z \in n^{-1} \mathbb{Z}_{0}^{d}$ and $n \geqslant 1$, and that $\sup _{n \geqslant 1}\left\|\Phi_{n}\right\|_{\infty}<\infty$ thanks to (1.7). Thus we have established that Assumption (C2) holds for every $\omega \in \Omega_{0}$. Therefore by Theorem 2.7, we get the conclusion for $\alpha=2$ in Theorem 1.2.

Next, we present the

\footnotetext{
${ }^{1}$ For reader's convenience, we present a proof of this fact in this footnote. Denote by $C_{\infty}\left(\mathbb{R}^{d}\right)$ the space of continuous functions on $\mathbb{R}^{d}$ that vanish at infinity, and $C_{\infty}^{2}\left(\mathbb{R}^{d}\right)$ the space of $C^{2}$-smooth functions on $\mathbb{R}^{d}$ that together with their derivatives up to second orders vanish at infinity. Observe that $C_{\infty}\left(\mathbb{R}^{d}\right)$ is a separable Banach space under the uniform norm $\|\cdot\|_{\infty}$. Denote by $\Phi$ the isometric map from $\left(C_{\infty}^{2}\left(\mathbb{R}^{d}\right),\|\cdot\|_{2, \infty}\right)$ into $\left(C_{\infty}\left(\mathbb{R}^{d}\right)^{1+d+d^{2}},\|\cdot\|_{\infty}\right)$ defined by $\Phi(f)=$ $\left(f, \nabla f, \nabla^{2} f\right)$. Note that $\Phi\left(C_{\infty}^{2}\left(\mathbb{R}^{d}\right)\right)$ is a closed subspace of $\left(C_{\infty}\left(\mathbb{R}^{d}\right)^{1+d+d^{2}},\|\cdot\|_{\infty}\right)$ and hence is separable under the norm $\|\cdot\|_{\infty}$. It follows that $\left(C_{\infty}^{2}\left(\mathbb{R}^{d}\right),\|\cdot\|_{2, \infty}\right)$ is separable. Let $\left\{g_{n} ; n \geqslant 1\right\}$ be a countable dense subsequence in $\left(C_{\infty}^{2}\left(\mathbb{R}^{d}\right),\|\cdot\|_{2, \infty}\right)$, and $\left\{\varphi_{k} ; k \geqslant 1\right\}$ a sequence of smooth functions with compact support on $\mathbb{R}^{d}$ so that $\varphi_{k}(x)=1$ for $|x| \leqslant k$ and $\varphi_{k}(x)=0$ for $|x| \geqslant k+1$ with $\sup _{k \geqslant 1}\left\|\varphi_{k}\right\|_{2, \infty}<\infty$. Then $\left\{g_{n} \varphi_{k} ; n, k \geqslant 1\right\}$ is a countable dense sequence in $\left(C_{c}^{2}\left(\mathbb{R}^{d}\right),\|\cdot\|_{2, \infty}\right)$.
} 
Proof of Proposition 1.3. By (1.9) and the continuity of $K$ in $\mathbb{R}_{\mathbf{0}}^{d}$, we have

$$
K(s z)=K(z), \quad z \in \mathbb{R}_{\mathbf{0}}^{d}, s>0 .
$$

Therefore, the function $K: \mathbb{R}_{\mathbf{0}}^{d} \rightarrow \mathbb{R}_{+}$can be viewed as a function defined on $\mathbb{S}^{d-1}$.

According to (1.9), for every integer $R \geqslant 1$,

$$
\lim _{n \rightarrow \infty} \sup _{x \in n^{-1} \mathbb{Z}^{d}:|x| \leqslant R} \frac{1}{\log (1+n)} \sum_{z \in \mathbb{Z}_{\mathbf{0}}^{d}:|z| \leqslant n}\left|\frac{z_{i} z_{j} \mathbb{E}[\kappa(n x, z)]}{|z|^{d+2}}-\frac{z_{i} z_{j} K(z / n)}{|z|^{d+2}}\right|=0 .
$$

Indeed, (1.9) implies that for any integer $R \geqslant 1$ and $\varepsilon \in(0,1)$,

$$
\lim _{n \rightarrow \infty} \sup _{x \in n^{-1} \mathbb{Z}^{d}:|x| \leqslant R} \frac{1}{\log (1+n)} \sum_{z \in \mathbb{Z}_{0}^{d}: n^{\varepsilon} \leqslant|z| \leqslant n}\left|\frac{z_{i} z_{j} \mathbb{E}[\kappa(n x, z)]}{|z|^{d+2}}-\frac{z_{i} z_{j} K(z / n)}{|z|^{d+2}}\right|=0 .
$$

On the other hand, it follows from the boundedness of $K$ and $\sup _{x \in \mathbb{Z}^{d}, z \in \mathbb{Z}_{\mathbf{0}}^{d}} \mathbb{E}[\kappa(x, z)]<\infty$ that for any integer $R \geqslant 1$ and $\varepsilon \in(0,1)$,

$$
\begin{aligned}
& \sup _{x \in n^{-1} \mathbb{Z}^{d}:|x| \leqslant R} \frac{1}{\log (1+n)} \sum_{z \in \mathbb{Z}_{0}^{d}:|z| \leqslant n^{\varepsilon}}\left|\frac{z_{i} z_{j} \mathbb{E}[\kappa(n x, z)]}{|z|^{d+2}}-\frac{z_{i} z_{j} K(z / n)}{|z|^{d+2}}\right| \\
& \leqslant \frac{\sup _{x \in \mathbb{Z}^{d}, z \in \mathbb{Z}_{0}^{d}} \mathbb{E}[\kappa(x, z)]+\|K\|_{\infty}}{\log (1+n)} \sum_{z \in \mathbb{Z}_{0}^{d}: \mid z n^{\varepsilon}} \frac{\left|z_{i}\right|\left|z_{j}\right|}{|z|^{d+2}} \\
& \leqslant c_{0} \frac{\varepsilon \log (1+n)}{\log (1+n)} \rightarrow c_{0} \varepsilon \quad \text { as } n \rightarrow \infty .
\end{aligned}
$$

Putting both estimates above together and letting $\varepsilon \rightarrow 0$, we get (3.13). Hence, in order to verify (1.8) it suffices to show

$$
\lim _{n \rightarrow \infty}\left|\frac{1}{\log (1+n)} \sum_{z \in \mathbb{Z}_{0}^{d}:|z| \leqslant n} \frac{z_{i} z_{j} K(z / n)}{|z|^{d+2}}-a_{i j}\right|=0, \quad 1 \leqslant i, j \leqslant d,
$$

where $a_{i j}=\int_{\mathbb{S}^{d-1}} K(\theta) \theta_{i} \theta_{j} d \theta$.

It follows from (3.12) that

$$
\begin{aligned}
\frac{1}{\log (1+n)} \sum_{z \in \mathbb{Z}_{0}^{d}:|z| \leqslant n} \frac{z_{i} z_{j} K(z / n)}{|z|^{d+2}} & =\frac{1}{n^{d} \log (1+n)} \sum_{z \in n^{-1} \mathbb{Z}^{d: 0<|z| \leqslant 1}} \frac{z_{i} z_{j} K(z)}{|z|^{d+2}} \\
& =\frac{1}{\log (1+n)} \sum_{z \in n^{-1} \mathbb{Z}^{d}: 0<|z| \leqslant 1} \int_{Q_{n}(z)} \frac{z_{i} z_{j} K(z)}{|z|^{d+2}} d y,
\end{aligned}
$$

where $Q_{n}(z):=\Pi_{1 \leqslant i \leqslant d}\left(z_{i}-1 / 2 n, z_{i}+1 /(2 n)\right]$ for $z:=\left(z_{1}, \cdots, z_{d}\right) \in n^{-1} \mathbb{Z}^{d}$. By the mean value theorem and the fact that $K$ is uniformly bounded, for any $z \in n^{-1} \mathbb{Z}^{d}$ with $\frac{\sqrt{d}}{n} \leqslant|z| \leqslant 1$ and any $y \in Q_{n}(z)$,

$$
\left|\frac{z_{i} z_{j} K(z)}{|z|^{d+2}}-\frac{y_{i} y_{j} K(y)}{|y|^{d+2}}\right| \leqslant c_{1}\left(\frac{1}{n\left(|z|-\frac{\sqrt{d}}{2 n}\right)^{d+1}}+\frac{\xi_{n}}{\left(|z|-\frac{\sqrt{d}}{2 n}\right)^{d}}\right)
$$

where

$$
\xi_{n}:=\sup _{0<|y|,|z| \leqslant 1,|y-z| \leqslant \frac{\sqrt{d}}{2 n}}|K(z)-K(y)| .
$$

Therefore,

$$
I(n):=\left|\frac{1}{\log (1+n)} \sum_{z \in n^{-1} \mathbb{Z}^{d}: 0<|z| \leqslant 1} \int_{Q_{n}(z)} \frac{z_{i} z_{j} K(z)}{|z|^{d+2}} d y-\frac{1}{\log (1+n)} \int_{\{1 / n \leqslant|y| \leqslant 1\}} \frac{y_{i} y_{j} K(y)}{|y|^{d+2}} d y\right|
$$




$$
\begin{aligned}
\leqslant & \frac{1}{n^{d} \log (1+n)} \sum_{z \in n^{-1} \mathbb{Z}^{d}:|z|<\frac{\sqrt{d}}{n}}\left|\frac{z_{i} z_{j} K(z)}{|z|^{d+2}}\right| \\
& +\frac{1}{\log (1+n)} \int_{\left\{\left(\frac{\sqrt{d}}{2 n} \wedge \frac{1}{n}\right) \leqslant|y| \leqslant \frac{3 \sqrt{d}}{2 n}\right\} \cup\left\{1 \leqslant|y| \leqslant 1+\frac{\sqrt{d}}{2 n}\right\}}\left|\frac{y_{i} y_{j} K(y)}{|y|^{d+2}}\right| d y \\
& +\frac{1}{n^{d} \log (1+n)} \sum_{z \in \mathbb{Z}^{d}: \frac{\sqrt{d}}{n} \leqslant|z| \leqslant 1} \int_{Q_{n}(z)}\left|\frac{z_{i} z_{j} K(z)}{|z|^{d+2}}-\frac{y_{i} y_{j} K(y)}{|y|^{d+2}}\right| d y \\
= & : I_{1}^{n}+I_{2}^{n}+I_{3}^{n} .
\end{aligned}
$$

It is clear that

and, by (3.15),

$$
I_{1}^{n} \leqslant \frac{c_{1}}{\log (1+n)}, \quad I_{2}^{n} \leqslant \frac{c_{2}}{\log (1+n)}
$$

$$
I_{3}^{n} \leqslant \frac{c_{1}}{n^{d} \log (1+n)} \sum_{k=[\sqrt{d}]}^{n} \sum_{z \in n^{-1} \mathbb{Z}^{d}:|z|=\frac{k}{n}}\left(\frac{n^{d}}{\left(k-\frac{\sqrt{d}}{2}\right)^{d+1}}+\frac{n^{d} \xi_{n}}{\left(k-\frac{\sqrt{d}}{2}\right)^{d}}\right) \leqslant c_{3}\left(\frac{1}{\log (1+n)}+\xi_{n}\right) .
$$

Since $K(\cdot)$ is a continuous function on $\mathbb{R}_{\mathbf{0}}^{d}$ and satisfies $(3.12), \lim _{n \rightarrow \infty} \xi_{n}=0$. Hence we deduce from the above estimates that

$$
\lim _{n \rightarrow \infty} I(n)=0
$$

This along with (3.14) gives us

$$
\begin{aligned}
& \lim _{n \rightarrow \infty}\left|\frac{1}{\log (1+n)} \sum_{z \in \mathbb{Z}_{0}^{d}:|z| \leqslant n} \frac{z_{i} z_{j} K(z / n)}{|z|^{d+2}}-\int_{\mathbb{S}^{d-1}} \theta_{i} \theta_{j} K(\theta) d \theta\right| \\
& =\lim _{n \rightarrow \infty}\left|\frac{1}{\log (1+n)} \int_{\{1 / n \leqslant|y| \leqslant 1\}} \frac{y_{i} y_{j} K(y)}{|y|^{d+2}} d y-\int_{\mathbb{S}^{d-1}} \theta_{i} \theta_{j} K(\theta) d \theta\right| \\
& =\lim _{n \rightarrow \infty}\left|\frac{1}{\log (1+n)} \int_{1 / n}^{1} r^{-1} \int_{\mathbb{S}^{d-1}} \theta_{i} \theta_{j} K(\theta) d \theta d r-\int_{\mathbb{S}^{d-1}} \theta_{i} \theta_{j} K(\theta) d \theta\right| \\
& =0,
\end{aligned}
$$

where in the second equality we used (3.12). In particular, we have verified that the limit in (1.8) exists with $a_{i j}=\int_{\mathbb{S}^{d-1}} \theta_{i} \theta_{j} K(\theta) d \theta$.

\section{EXTENSIONS AND REMARKS}

4.1. Extensions. From the proof for the case $\alpha \in(0,2)$ in Theorem 1.2, one can see that, at the expense of a higher moments condition on $\kappa(x, z)$, it is possible to relax the independence Assumption (A0) to a block independence condition that

$$
\left\{\left\{\kappa(x, z): z \in \mathbb{Z}_{+, *}^{d} \text { with }|z|=r\right\}: x \in \mathbb{Z}^{d}, r \geqslant 1\right\} \text { are independent. }
$$

When $\alpha \in(0,1)$, since $1 /|z|^{d+\alpha}$ is integrable over the unit ball $B(0,1)$ in $\mathbb{R}^{d}$, we can drop the balanced condition (1.1) on $\kappa(x, z)$. In this case, Theorem 1.2(i) holds with Assumption (A0) and Assumption (A1)(i) being replaced by

Assumption (A0*) $\left\{\kappa(x, z): x \in \mathbb{Z}^{d}, z \in \mathbb{Z}_{\mathbf{0}}^{d}\right\}$ is a sequence of independent non-negative random variables.

Assumption $\left(\mathbf{A 1}^{*}\right)(\mathrm{i}) d>2-2 \alpha$ and there is some $p>\max \left\{\frac{2(d+1)}{d}, \frac{d+1}{1-\alpha}\right\}$ such that

$$
\sup _{x \in \mathbb{Z}^{d}, z \in \mathbb{Z}_{\mathbf{0}}^{d}} \mathbb{E}\left[\kappa(x, z)^{p}\right]<\infty .
$$


Indeed, as is shown in the proof of [6, Proposition 5.6], we know that Assumption $\left(\mathbf{A} \mathbf{0}^{*}\right)$ and $\left(\mathbf{A} \mathbf{1}^{*}\right)(\mathrm{i})$ together imply Assumption (B1*). Hence the conclusion of Theorem 1.2(i) still holds by following the proof of Theorem 1.2(i) and by using Theorem 2.6.

4.2. Remarks. Now suppose $\alpha>2$. We consider the following two conditions.

Assumption (D1).

$$
\sup _{x \in \mathbb{Z}^{d}} \sum_{z \in \mathbb{Z}_{0}^{d}} \frac{\kappa(x, z)}{|z|^{d+\alpha-2}}<\infty
$$

Assumption (D2). For any $n \geqslant 1$, there exists a function $\Phi_{n}(x, z): n^{-1} \mathbb{Z}^{d} \times n^{-1} \mathbb{Z}_{\mathbf{0}}^{d} \rightarrow(0, \infty)$ with $\Phi_{n}(x, z)=\Phi_{n}(x,-z)$ for all $x \in n^{-1} \mathbb{Z}^{d}$ and $z \in n^{-1} \mathbb{Z}_{0}^{d}$, so that $\sup _{n \geqslant 1}\left\|\Phi_{n}\right\|_{\infty}<\infty$, and for any integer $R \geqslant 1$ and every $f \in C_{c}^{2}\left(\mathbb{R}^{d}\right)$,

$$
\lim _{n \rightarrow \infty} n^{2} \sup _{\substack{x \in \mathbb{Z}^{d}: \\|x| \leqslant n R}}\left|\sum_{z \in \mathbb{Z}_{\mathbf{0}}^{d}}\left(f\left(\frac{x+z}{n}\right)-f\left(\frac{x}{n}\right)\right)\left(\frac{\kappa(x, z)-\Phi_{n}(x / n, z / n)}{|z|^{d+\alpha}}\right)\right|=0,
$$

and that for any integer $R \geqslant 1$ and $1 \leqslant i, j \leqslant d$,

$$
\left.\lim _{n \rightarrow \infty} \sup _{x \in n^{-1} \mathbb{Z}^{d}:|x| \leqslant R}\left|\sum_{z \in \mathbb{Z}_{\mathbf{0}}^{d}} z_{i} z_{j} \Phi_{n}(x, z / n) /\right| z\right|^{d+2}-a_{i j} \mid=0
$$

for some constant matrix $A:=\left(a_{i j}\right)_{1 \leqslant i, j \leqslant d}$ on $\mathbb{R}^{d}$.

We can establish the following theorem by a similar argument as that of Theorem 2.7.

Theorem 4.1. Let $\alpha>2$. Assume that the balanced condition (1.1), and Assumptions (D1) and (D2) hold. Then the conclusion of Theorem 2.7 holds.

However, we are unable obtain the corresponding result of Theorem 1.2 for $\alpha>2$ in random environments under the Assumption (A0) and (1.8) (but with $|z|^{-d-\alpha}$ in place of $|z|^{-d-2}$ and without the $(\log n)^{-1}$ factor). It appears that the Borel-Cantelli argument to verify Assumptions (D1) and (D2) fails in this setting. This is because when $\alpha>2$, the behavior of the limiting process for the scaled processes is not determined merely by the expectation of random coefficients $\kappa(x, z)$ as in the the case of NNBRWs in random environments (see, for instance, [5]).

Acknowledgements. We thank the anonymous referee for valuable comments. The research of Xin Chen is supported by the National Natural Science Foundation of China (Nos. 11501361 and 11871338). The research of Zhen-Qing Chen is partially supported by Simons Foundation Grant 520542 and a Victor Klee Faculty Fellowship at UW. The research of Takashi Kumagai is supported by JSPS KAKENHI Grant Number JP17H01093 and by the Alexander von Humboldt Foundation. The research of Jian Wang is supported by the National Natural Science Foundation of China (Nos. 11831014 and 12071076), the Program for Probability and Statistics: Theory and Application (No. IRTL1704) and the Program for Innovative Research Team in Science and Technology in Fujian Province University (IRTSTFJ).

\section{REFERENCES}

[1] Aldous, D.: Stopping times and tightness, Ann. Probab., 6, 1978, 335-340.

[2] Bass, R.F. and Tang, H.: The martingale problem for a class of stable-like processes, Stochastic Process. Appl., 119, 2009, 1144-1167.

[3] Bercu, B., Delyon, B. and Rio, E.: Concentration Inequalities for Sums and Martingales, Springer, 2015.

[4] Berger, N., Cohen, M., Deuschel, J.-D. and Guo, X.: An elliptic Harnack inequality for random walk in balanced environments, arXiv:1807.03531.

[5] Berger, N. and Deuschel, J.-D.: A quenched invariance principle for non-elliptic random walk in i.i.d. balanced random environment, Probab. Theory Related Fields, 158, 2014, 91-126. 
[6] Chen, X., Kumagai, T. and Wang, J.: Random conductance models with stable-like jumps: quenched invariance principle, to appear in Ann. Appl. Probab., arXiv:1805.04344.

[7] Chen, Z.-Q. and Zhang, X.: Uniqueness of stable-like processes, arXiv:1604.02681.

[8] Deuschel, J.-D. and Guo, X.: Quenched local central limit theorem for random walks in a time-dependent balanced random environment, arXiv:1710.05508.

[9] Deuschel, J.-D., Guo, X. and Ramirez, A.: Quenched invariance principle for random walk in time-dependent balanced random environment, Ann. Inst. H. Poincaré Probab. Statist., 54, 2018, 363-384.

[10] Guillen, N. and Schwab, R.W.: Aleksandrov-Bakelman-Pucci type estimates for integro-differential equations, Arch. Ration. Mech. Anal., 206, 2012, 111-157.

[11] Guo, X. and Zeitouni, O.: Quenched invariance principle for random walks in balanced random environment, Probab. Theory Related Fields, 152, 2012, 207-230.

[12] Lawler, G.: Weak convergence of a random walk in a random environment, Comm. Math. Phys., 87, 1982, 81-87.

[13] Papanicolaou, G.C. and Varadhan, S.R.S.: Diffusions with random coefficients, in Statistics and Probability: Essays in Honor of Rao, C.R., 547-552, North-Holland, Amsterdam, 1982.

[14] Peng, J.: Uniqueness in law for stable-like processes of variable order, to appear in J. Theoret. Probab., arXiv:1802.01151.

Xin Chen: Department of Mathematics, Shanghai Jiao Tong University, 200240 Shanghai, P.R. China. chenxin217@sjtu.edu.cn

Zhen-Qing Chen: Department of Mathematics, University of Washington, Seattle, WA 98195, USA. zqchen@uw. edu

Takashi Kumagai: Research Institute for Mathematical Sciences, Kyoto University, Kyoto 606-8502, Japan. kumagai@kurims.kyoto-u.ac.jp

Jian Wang: College of Mathematics and Informatics \& Fujian Key Laboratory of Mathematical Analysis and Applications (FJKLMAA) \& Center for Applied Mathematics of Fujian Province (FJNU), Fujian Normal University, 350007 Fuzhou, P.R. China. jianwang@f jnu.edu.cn 